\title{
Old and Young X-ray Point Source Populations in Nearby Galaxies
}

\author{
Edward J. M. Colbert, Timothy M. Heckman, Andrew F. Ptak, and David K. Strickland \\ Johns Hopkins University, Department of Physics and Astronomy, Homewood Campus, \\ 3400 North Charles Street, Baltimore, MD 21218 \\ and \\ Kimberly A. Weaver \\ Laboratory for High Energy Astrophysics, Code 662, NASA/GSFC, Greenbelt, MD 20771; \\ and Johns Hopkins University, Department of Physics and Astronomy, Homewood \\ Campus, 3400 North Charles Street, Baltimore, MD 21218
}

\begin{abstract}
We have analyzed Chandra ACIS observations of 32 nearby spiral and elliptical galaxies and present the results of $1441 \mathrm{X}$-ray point sources that were detected in these galaxies. The total point-source X-ray $(0.3-8.0 \mathrm{keV})$ luminosity $\mathrm{L}_{X P}$ is well correlated with the B-band, K-band, and FIR+UV luminosities of spiral host galaxies, and is well correlated with the B-band and K-band luminosities for elliptical galaxies. This suggests an intimate connection between $\mathrm{L}_{X P}$ and both the old and young stellar populations, for which $\mathrm{K}$ and FIR+UV luminosities are reasonable proxies for the galaxy mass $M$ and star-formation rate $S F R$. We derive proportionality constants $\alpha=1.3 \times 10^{29} \mathrm{erg} \mathrm{s}^{-1} \mathrm{M}_{\odot}^{-1}$ and $\beta=0.7 \times 10^{39} \mathrm{erg} \mathrm{s}^{-1}\left(\mathrm{M}_{\odot} \mathrm{yr}^{-1}\right)^{-1}$, which can be used to estimate the old and young components from $M$ and $S F R$, respectively. The cumulative Xray luminosity functions for the point sources have significantly different slopes. For the spiral and starburst galaxies, $\gamma \approx 0.6-0.8$, and for the elliptical galaxies, $\gamma \approx 1.4$. This implies that the most luminous point sources - those with $L_{X} \gtrsim$ $10^{38} \mathrm{erg} \mathrm{s}^{-1}$ - dominate $L_{X P}$ for the spiral and starburst galaxies. Most of the point sources have X-ray colors that are consistent with soft-spectrum (photon index $\Gamma \sim 1-2$ ) low-mass X-ray binaries, accretion-powered black-hole high-mass (BH HMXBs), or Ultra-Luminous X-ray sources (ULXs a.k.a. IXOs). We rule out hard-spectrum neutron-star HMXBs (e.g. accretion-powered X-ray pulsars) as contributing much to $\mathrm{L}_{X P}$. Thus, for spirals, $\mathrm{L}_{X P}$ is dominated by ULXs and $\mathrm{BH}$ HMXBs. We find no discernible difference between the X-ray colors of ULXs
\end{abstract}


$\left(\mathrm{L}_{X} \geq 10^{39} \mathrm{erg} \mathrm{s}^{-1}\right)$ in spiral galaxies and point sources with $\mathrm{L}_{X} \approx 10^{38}-10^{39}$ $\mathrm{erg} \mathrm{s}^{-1}$. We estimate that $\gtrsim 20 \%$ of all ULXs found in spirals originate from the older (pop II) stellar populations, indicating that many of the ULXs that have been found in spiral galaxies are in fact pop II ULXs, like those in elliptical galaxies. We find that $\mathrm{L}_{X P}$ depends linearly (within uncertainties) on both $M$ and $S F R$, for our sample galaxies $\left(M \lesssim 10^{11} \mathrm{M}_{\odot}\right.$ and $\left.S F R \lesssim 10 \mathrm{M}_{\odot} \mathrm{yr}^{-1}\right)$.

Subject headings: galaxies: elliptical — galaxies: fundamental parameters - galaxies: spiral - galaxies: starburst - X-rays: galaxies - X-rays: binaries

\section{Introduction}

As the resolution and sensitivity of X-ray imaging detectors continues to improve, it becomes more and more feasible to study individual X-ray point sources in external galaxies. The point-spread function (PSF) of the Einstein IPC imaging spectrometer $\left(\sim 1^{\prime}\right)$ was typically too poor to distinguish emission from individual point sources in galaxies with distances $\gtrsim 2 \mathrm{Mpc}$, and, for more nearby galaxies, it was usually only sensitive to very luminous sources with $\mathrm{L}_{X} \gtrsim 10^{38} \mathrm{erg} \mathrm{s}^{-1}$. The ROSAT PSPC (PSF $\sim 20^{\prime \prime}$ ) offered some improvement, and some nearby galaxies could be studied in the soft $(0.2-2.4 \mathrm{keV})$ band. Fabbiano (1989) gives a comprehensive review of X-ray sources in nearby galaxies in the pre-Chandra era. The Chandra ACIS instrument has a tremendous improvement in both spatial resolution $\left(\mathrm{PSF} \sim 1^{\prime \prime}\right)$ and sensitivity, and covers the bandpass $0.2-8.0 \mathrm{keV}$. We are now able to study the properties of individual point sources in galaxies out to $\sim 20 \mathrm{Mpc}$, for which $\mathrm{L}_{X} \approx 10^{38}$ $\mathrm{erg} \mathrm{s}^{-1}$ sources are detected in a reasonable $(50 \mathrm{ks})$ exposure time. Although the bright X-ray sources in the Milky Way (MW) are easily studied, many of the MW point sources are viewed through the obscuring galaxy disk, so one must correct for completeness when doing statistical work. Furthermore, in order to study how properties of classes of point sources correlate with host galaxy properties, it is imperative to study large samples of different types of galaxies. We are now able to study properties of low-mass X-ray binaries (LMXBs), high-mass X-ray binaries (HMXBs), and Ultra-Luminous X-ray sources $\left(\mathrm{L}_{X} \geq 10^{39} \mathrm{erg} \mathrm{s}^{-1}\right.$; ULXs, a.k.a. Intermediate-luminosity X-ray Objects [IXOs]) in large samples of external galaxies with Chandra.

Hard (kT $\sim 2-10 \mathrm{keV}) \mathrm{X}$-ray emission is often present in the halos of elliptical galaxies, and sometimes also in starburst galaxy nuclei (e.g. Moran \& Lehnert 1997 and Ptak et al. 1997). ASCA was not able to resolve or diagnose the origin of the hard emission because of its poor spatial resolution (PSF $\sim 1^{\prime}$ ). However, Chandra has shown that X-ray point sources in spiral and elliptical galaxies often emit a large fraction of the total hard X-ray 
luminosity. We can now address some important questions with large surveys of galaxies with Chandra. How do the X-ray point source populations of all of the different types of X-ray binaries (XRBs) and ULXs depend on the properties of the host galaxy? What is the exact connection between starbursts (or young star formation) and XRB creation and evolution? How does the host galaxy environment affect the evolution of the sources? Is there a connection between the XRB population and the ULX population? Results from the many Chandra observations of spiral and elliptical galaxies already show important progress in many of these areas of study (e.g., see review articles by Irwin et al. 2002a, Roberts et al. 2001, and Miller \& Colbert 2003, and references therein).

Here we present results from a study of the properties of the X-ray point source population in a sample of 5 Merger and Irregular galaxies and 18 spiral galaxies, many of which have high star formation rates (SFRs), and 9 elliptical galaxies, which typically have negligible SFRs. We have analyzed the ACIS data for these 32 galaxies in exactly the same way. Thus, results from our processed data will not be affected by different analysis techniques, as might have been the case if results from already published work on individual objects were used for the present study, such as tabulations listed in the many papers on Chandra observations of galaxies (e.g, Eracleous et al. 2002, Swartz et al. 2003, Sarazin, Irwin \& Bregman 2001, and Irwin, Sarazin \& Bregman 2002b). Since our calibration data, analyses software, or filtering criteria may be different (see section 3.1), our results (e.g. number of sources, count rates, fluxes, and luminosities) may differ slightly from those already reported in the literature.

In section 2, we describe the galaxy sample. Data reduction techniques are discussed in section 3 and results are given in section 4 . We discuss the implications of the results in section 5, and summarize in section 6 .

\section{Description of Data and Galaxy Sample}

Data for twenty-seven of the galaxies in the sample were selected randomly from the nearby $(\mathrm{D}<30 \mathrm{Mpc}) \mathrm{NGC}$ and Messier galaxies that were were in the public Chandra archives on September 3, 2001. Proprietary data for galaxies NGC 3079, NGC 3628, NGC 5253 and NGC 4449 were also added. When the Chandra observation of the very nearby elliptical galaxy NGC 3379 became public in February 2002, we added it as well.

Since most of the data were taken from the Chandra archives and therefore the sample is already biased toward galaxies that are historically interesting in the X-ray band, there is no obvious way to select a 'complete,' unbiased sample. Therefore, our goal was to 
select $\sim 30$ galaxies of all morphological types, and with a wide range in "starburst power," since we are interested in studying how the X-ray point source population varies with host galaxy properties. Since the XASSIST software (see section 3.1 ) typically requires $\sim 1$ day of computer processing (or re-processing) per dataset, a sample size of $\sim 30$ galaxies was both feasible, and large enough for useful statistical work. We imposed the restriction that all of the sample galaxies must have X-ray luminosity sensitivity t $/ \mathrm{D}^{2}>0.044 \mathrm{ks} \mathrm{Mpc}^{-2}$, where $t$ is the exposure time after filtering (see Table 1 ), and $D$ is the distance to the galaxy (Table 2 ). This corresponds to a luminosity sensitivity of $\approx 10^{38} \mathrm{erg} \mathrm{s}^{-1}$ for a back-illuminated (BI) CCD, such as CCD 7.

Since our intent is to study the relationship between the X-ray point source populations and the properties of the stellar component of the host galaxies (and not the active supermassive black holes [SMBHs] and their possible connection with intermediate-mass black holes [IMBHs], for example), we intentionally omitted the three powerful active galaxies NGC 1068, NGC 4151, and M87. Although the peculiar elliptical galaxy Cen A has an AGN with a radio jet, like M87, it is the only "elliptical" galaxy with significant star formation, so we decided to keep it in our sample. LINERs and weak Seyferts were not omitted intentionally (see column 4 of Table 2). The composite starburst/AGN galaxy NGC 4945 was also kept.

Our final sample of 32 galaxies (see Table 1) includes 28 galaxies from the archive, and proprietary data for four galaxies (NGC 3079, NGC 3628, NGC 4449, and NGC 5253).

In Table 2, we list some host galaxy properties that are related to the stellar content of the galaxy, in particular properties that are related to the star-formation rate (SFR) and the stellar mass (M). The far-infrared (FIR) and far-ultraviolet luminosities are proxies for the $\mathrm{SFR}$, while the B-band and $\mathrm{K}_{s}$-band luminosities are proxies for the mass (e.g. Kennicutt 1998, Bell \& de Jong 2001). Note that the galaxies in our sample span a wide range in both morphological type, and in SFR/M (see $\mathrm{L}_{F I R} / \mathrm{L}_{B}$ or $\mathrm{L}_{F I R+U V} / \mathrm{L}_{K}$; Table 2).

For galaxies with recessional velocities $<1000 \mathrm{~km} \mathrm{~s}^{-1}$, as listed in the Third Reference Catalog of Bright Galaxies (RC3; de Vaucouleurs et al. 1991), distances were taken directly from Tully (1988). We used 21-cm recessional velocities when available, otherwise optical recessional velocities. For galaxies with recessional velocities $\geq 1000 \mathrm{~km} \mathrm{~s}^{-1}$, we calculated the distance using $\mathrm{H}_{0}=75 \mathrm{~km} \mathrm{~s}^{-1} \mathrm{Mpc}^{-1}$. The distances to NGC 4038 and NGC 5094 were used for X-ray sources in NGC 4038/9 and NGC 5194/5, respectively. 


\section{Data Reduction}

\subsection{X-ray Data}

All of the public ACIS data were retrieved from the SAO Chandra archives. All of the data were reprocessed and reduced using a modified version of the XASSIST v0.757 (Ptak \& Griffiths 2003) scripts, LHEASOFT v5.2, and CIAO v2.2.

Level-1 event files were reprocessed to level-2 event files with CIAO, using XASSIST. XASSIST uses the basic data reduction steps recommended by the CXC "threads." The optional 0.5 pixel position randomization was not performed. After this reprocessing step, each CCD is treated as a different detector, and individual CCD data are processed separately. For data processing, PI values were constrained to be in the range 14-548, corresponding to the energy range $0.2-8.0 \mathrm{keV}$, although fluxes and counts for X-ray colors were computed from the data in the $0.3-8.0 \mathrm{keV}$ range.

The CIAO WAVDETECT source detection routine was then used on the reprocessed level-2 event data to produce a preliminary list of point sources. For all sources with more than 10 net counts, we further tested the robustness of the detection using a CPU-intensive 2D Gaussian image-fitting algorithm. If a reasonable fit to the image was obtained with a Gaussian (source) plus sloping plane (background) model, we were able to estimate a more accurate count rate, and better estimates of the major and minor axes of the source. If the ratio of the major to minor axis was larger than 2.0, the source was initially flagged as 'extended.' Likewise, if the size of the major or minor axes was larger than the PSF (at that off-axis angle), the source was also flagged as 'extended.' Sources detected at S/N < 2.0 were rejected, as were sources with fitted Gaussian sizes too small to be consistent with the ACIS PSF.

We then filtered only those sources inside the galaxy major and minor $R_{25}$ ellipse, as listed in RC3. Each source was then individually inspected on-screen, and re-classified as 'point-like' or 'extended.' Any X-ray sources that were within 5" of the galaxy nucleus (as listed in NED) were considered potential active nuclei and were flagged for further examination. After checking each case by hand, a single nuclear X-ray source was omitted in each of the following seven galaxies: NGC 3079, NGC 4258, NGC 4374, NGC 4579, NGC 4945, NGC 5128, and NGC 5194. The 'jet' sources in NGC 4258 and Cen A were also rejected. We also rejected all of the artificial X-ray sources that were produced along the readout column of high count-rate sources (NGC 4579 and NGC 5128). After screening, we found a total of 1441 point sources in 32 galaxies (see Table 1 and Appendix). As mentioned in section

1, our data reduction techniques and filtering methods may be different from those of other workers, so our point source lists may also differ. 
The major and minor axes for elliptical source regions were determined directly from the Gaussian fitting, or from WAVDETECT if the Gaussian fitting was not performed, or failed. Local annular regions surrounding the source, and centered on the source position, were used for background. In crowded regions, when other source regions overlapped with the background region, the contaminating part(s) of the background region were omitted.

These source and background regions were used directly to compute counts in three energy ranges, for hardness ratios (see Table A1). X-ray fluxes in the $0.3-8.0 \mathrm{keV}$ band were determined for each source using the net count rates from the Gaussian fitting algorithm when available. Otherwise, the count rates from WAVDETECT were used. We converted the count rates to fluxes and luminosities using a simple power-law model with $\Gamma=1.8$, and the Galactic Hydrogen columns and distances listed in Table 2. Auxiliary Response Files (ARFs) generated using CIAO PSEXTRACT were used to compute fluxes, when they were available. Otherwise, on-axis ARF files were used.

We can estimate the uncertainties in our X-ray point-source luminosities using a simple absorbed power-law model in XSPEC. For a typical Galactic column of $\mathrm{N}_{H}=3 \times 10^{20}$ $\mathrm{cm}^{-2}$, the flux/count-rate ratio varies by $5-10 \%$ when $\Gamma$ varies by $\pm 0.2(\Gamma=1.6-2.0)$. If $\Gamma$ varies by $\pm 0.7(\Gamma=1.1-2.5)$, the ratio varies by $30-40 \%$. Uncertainties in the absorption column are also important. Compared with a model with $\mathrm{N}_{H}=3 \times 10^{20} \mathrm{~cm}^{-2}$, a more higly absorbed model with $\mathrm{N}_{H}=3 \times 10^{21} \mathrm{~cm}^{-2}$ has flux/count-rate ratios $5-25 \%$ higher. This would cause our quoted luminosities to underestimate the true luminosities. For an highly-absorbed source with $\mathrm{N}_{H}=3 \times 10^{22} \mathrm{~cm}^{-2}$, the correction is severe $(\approx 100-190 \%)$. Thus, unless the source is highly absorbed, our estimates of the observed X-ray luminosities are within $\approx 30-40 \%$ of their actual value.

The total galaxy point-source X-ray luminosity $\mathrm{L}_{X P}$ was then computed by summing all of the individual X-ray luminosities for each of the point sources (see Tables A1 and 3). We list in Table 3 the fraction of $\mathrm{L}_{X P}$ at $\mathrm{L}_{X} \geq 10^{38} \mathrm{erg} \mathrm{s}^{-1}$, and the fraction at $\mathrm{L}_{X} \geq 10^{39}$ $\operatorname{erg~s}^{-1}$ (i.e., the ULXs). We also list the fraction of the total number of sources, and the total number of sources in these two high-luminosity ranges. The relatively small scatter in the ratio of $\mathrm{L}_{X P}$ to $\mathrm{L}_{K}$ in the last column of Table 3 shows the strong correlation between the stellar light and the X-ray light. As discussed in section 3.2, we believe our values of $\mathrm{L}_{X P}$ approximate the actual total point source luminosity within $\sim 20 \%$ for most of our galaxies. The exceptions are M82, NGC 253, and the elliptical galaxies, for which we estimate an uncertainty of $40 \%$.

Hardness ratios were computed for all sources (Table A1), but we used only the sources with $\geq 20$ net counts for our analyses. A total of 1017 sources met this criterion. Most (810) of these sources were detected on BI CCD 7. One additional source was detected on 
BI CCD5, and the rest of the sources were detected on front-illuminated (FI) CCDs (0, 1, $2,3$ and 6$)$.

We selected three energy bands for computing hardness ratios: $\mathrm{S}$ (soft, $0.3-1.0 \mathrm{keV}$ ), $\mathrm{M}$ (medium, 1.0-2.0 keV), and $\mathrm{H}$ (hard, 2.0-8.0 keV). Counts were extracted directly from the source and background event files described above. Hardness ratios of the form $\mathrm{HR}=$ $(\mathrm{C} 2-\mathrm{C} 1) /(\mathrm{C} 2+\mathrm{C} 1)$ were computed, where $\mathrm{C} 1$ and $\mathrm{C} 2$ are the net counts in the lower and higher energy bands, respectively. Hardness ratios from sources detected on the FI CCDs were transformed to the corresponding ratio for BI CCDs using effective area (EA) curves from the $\mathrm{CXC}$ website ${ }^{1}$. Corrections to the hardness ratio were obtained by estimating the correction to the counts in each energy band. For each of the sources on the FI CCDs, we first fit a power-law model to spectra in each energy band. We then computed the correction factor $f$ to the FI counts using the best-fit photon index for that band $\Gamma_{b a n d}$ and the equation

$$
f_{\text {band }}=\frac{\int_{\text {band }} E^{-\Gamma_{\text {band }}} E A(B I) d E}{\int_{\text {band }} E^{-\Gamma_{\text {band }}} E A(F I) d E} .
$$

The corrected counts are then $\mathrm{C} 1^{\prime}=f_{1} \mathrm{C} 1$ and $\mathrm{C} 2^{\prime}=f_{2} \mathrm{C} 2$, where $f_{1}$ and $f_{2}$ are the correction factors for the lower and high energy bands, respectively. Corrected hardness ratios $\mathrm{HR}^{\prime}$ were then computed from $\operatorname{HR}^{\prime}=(\phi+\mathrm{HR}) /(1+\phi \mathrm{HR})$, where $\phi=\left(1-f_{1} / f_{2}\right) /\left(1+f_{1} / f_{2}\right)$. Corrected hardness ratios for the sources are listed in Table A1.

\subsection{Unresolved X-ray Point Sources and Diffuse Hard X-ray Emission}

In order to test the accuracy of $\mathrm{L}_{X P}$ due to the omission of blended point sources, the erroneous addition of spurious point sources, and "compact" clumps of diffuse emission, we computed the total hard $(2.0-8.0 \mathrm{keV})$ counts from the individual point sources and compared with the total hard counts from the images within the $\mathrm{R}_{25}$ ellipse. Hard counts are expected only for point sources (e.g., XRBs) and diffuse hard X-ray emission, since the AGN and jet sources were omitted. In general, the hard counts for each method were consistent within $\sim 20 \%$, which is typical of the uncertainty in the total hard counts due to the large number of background counts in the ellipse. For M82 and NGC 253, there were excess hard counts, either in diffuse hard emission, or blended "unresolved" point sources (both are edge-on spiral galaxies). We also found evidence for excess hard counts in the elliptical galaxies. This is expected, since they are well known to have diffuse hard X-ray

\footnotetext{
${ }^{1}$ URL http://asc.harvard.edu/cal/Acis/Cal_prods/effarea/4_99/, EA * QE * filt.trans. curve files files orbit_i.dat $[\mathrm{FI}]$ and orbit_s.dat $[\mathrm{BI}]$
} 
emission. The diffuse hard emission in elliptical galaxies could be from multiple unresolved XRBs, or could be emission from diffuse, hot gas (e.g., Irwin et al. 2002b and references therein).

\subsection{Host Galaxy Luminosities}

As mentioned above, we calculated total luminosities from the galaxies in four separate energy bands: FIR, NIR $\mathrm{K}_{s}$-band, optical B-band, and the far-ultraviolet (FUV). All fluxes were converted to luminosities using the distances listed in Table 2. Far-infrared fluxes over the $40-120 \mu \mathrm{m}$ band were derived from the $60 \mu \mathrm{m}$ and $100 \mu \mathrm{m}$ IRAS fluxes using the method of Fullmer \& Lonsdale (1989). Near-infrared $\mathrm{K}_{s}$-band spectral fluxes $\mathrm{F}_{\lambda}$ were calculated from $20 \mathrm{mag} \operatorname{arcsec}^{-2}$ isophotal magnitudes listed in the 2MASS Large Galaxy Atlas (Jarrett et

al. 2003). Optical B-band spectral fluxes $\mathrm{F}_{\lambda}$ were calculated from $\mathrm{B}_{T}^{0}$ magnitudes (or $\mathrm{m}_{B}^{0}$ magnitudes for NGC 4038/9) listed in RC3. For NGC 4038/9 and NGC 5194/5, the total spectral flux was calculated using the sum of $\mathrm{F}_{\lambda}$ for each galaxy. In order to quote all luminosities in roughly the same size band, we have calculated $\mathrm{L}_{K}$ and $\mathrm{L}_{B}$ as $\lambda \mathrm{L}_{\lambda}$.

Far-ultraviolet fluxes were estimated using SED-fitting to broad-band total optical magnitudes (usually UBVRI, but in some cases UBVR or UBV), from Prugniel \& Heraudeau (1998). We experimented with a wide range of different SED databases, and obtained the best results using the observationally-based SEDs of Kinney \& Calzetti (Kinney et al. 1996, Calzetti et al. 1994) and the simulated HYPERZ SEDs (cf. Bolzonella et al. 2000). There was generally good agreement between our estimated FUV fluxes and published measurements (e.g., Rifatto et al. 1995, Marcum et al. 2001). The best-fit SEDs were then integrated from 1500-3500 $\AA$ to obtain the FUV flux. We list the B, K, FIR and FUV luminosities for each galaxy in Table 2 .

\section{Results}

\subsection{Simple Correlations of Total X-ray Point Source Luminosity with Host Galaxy Properties}

In Figure 1, we plot the total point-source $\mathrm{X}$-ray luminosity $\mathrm{L}_{X P}$ against the optical B-band, NIR K-band, FIR and FIR+FUV luminosities of the host galaxy. As noted by Fabbiano et al. (1988), both spiral and elliptical galaxies show a good correlation between the X-ray and B-band luminosity, suggesting that the X-ray luminosity is directly related to the number (or mass) of stars in the galaxy. The NIR K-band luminosity is a more accurate 
measure of the galaxy stellar mass and we prefer to use it for a proxy for the stellar mass instead of the B-band luminosity.

For all 32 galaxies in our sample, we find that $\mathrm{L}_{K}$ is slightly better correlated with $\mathrm{L}_{X P}$ (Pearson correlation coefficient $\left.\mathrm{r}=0.93\right)$ than is $\mathrm{L}_{B}(\mathrm{r}=0.90)$. The best fit yields $\mathrm{L}_{K} \propto$ $\mathrm{L}_{X P}^{0.97}$. When elliptical or spiral galaxies are considered separately, for both $B$ and $K$, the correlation is quite strong, with $r \approx 0.9$ (although $r \approx 0.8$ for $B$ and the spirals).

The FIR luminosity, which is an approximate measure of current star formation in latetype spirals and starburst galaxies, is only correlated $(r>0.50)$ with $\mathrm{L}_{X P}$ for the Merger/Irr and Spiral galaxies. When all of the galaxies are considered, there is no correlation between $\mathrm{L}_{F I R}$ and $\mathrm{L}_{X P}(r \approx 0.3)$. For ellipticals only, we find $r \approx 0.1$. The correlation is better $(r=0.68)$ for the spiral galaxies. We find the best correlation $(r=0.93)$ for the Merger/Irr galaxies, which, in general, have large SFR/M ratios (Table 2, columns 9 and 10). This suggests that a significant fraction of $\mathrm{L}_{X P}$ in high $\mathrm{SFR} / \mathrm{M}$ galaxies is due to current star formation, whereas in low-to-moderate SFR/M galaxies, much of $\mathrm{L}_{X P}$ could be due to the older population of X-ray sources. The correlation between SFR indicators and the total X-ray luminosity has been well noted in the literature (e.g. Helfand \& Moran 2001, Ranalli et al. 2003, Grimm et al. 2003). Here, we show specifically that the point-source X-ray luminosity $\mathrm{L}_{X P}$ is also well correlated with $S F R$ (and $M$ ).

Since some of the light from young, massive stars escapes directly from the galaxy as UV radiation, we use $\mathrm{L}_{F I R+U V}\left(\mathrm{~L}_{F I R}+\mathrm{L}_{F U V}\right)$ as a more accurate proxy for the current SFR. As with $\mathrm{L}_{F I R}$, when all galaxies are considered, there is only a weak correlation $(r \approx$ $0.5)$ between $\mathrm{L}_{F I R+U V}$ and $\mathrm{L}_{X P}$. We see a stronger correlation for the Merger/Irr and Spiral galaxies $(\mathrm{r}=0.94$ and 0.69 , respectively). For ellipticals only, there is absolutely no correlation $(r \approx 0.01)$.

A careful inspection of the $\mathrm{L}_{X P}-\mathrm{L}_{K}$ scatter plot in Figure 1 (upper right) reveals that the Merger/Irr and Spiral galaxies are systematically offset toward larger $\mathrm{L}_{X P}$, when compared to the Elliptical galaxies. This merely illustrates that there is a significant component of the point-source X-ray luminosity that is due to current star formation and is not directly related to the galaxy stellar mass. We discuss this effect further in section 5 . 


\subsection{X-ray Point Source Luminosity Functions}

In Figures 2 and 3, we show the cumulative point source X-ray Luminosity Functions (XLFs) $\mathrm{N}(>\mathrm{L})$ for all of the points sources from each of the 32 datasets. We list the slope ${ }^{2}$ $\gamma=-\mathrm{d} \log \mathrm{N}(>\mathrm{L}) / \mathrm{d} \log \mathrm{L}$ for each of the galaxies in Table 4. For the Merger/Irr group, the mean and standard deviation for $\gamma=0.65 \pm 0.16$, with the most prominent outlier being the dwarf galaxy NGC 5253 with $\gamma \approx 0.9$ (see Table 4). The spirals have very similar slopes: $\gamma=$ $0.79 \pm 0.24$. The luminosity functions of the elliptical galaxies, however, have steeper slopes: $\gamma=1.41 \pm 0.38$. These differences in XLF slopes has been known for some time (e.g., Primini et al. 1993, Kilgard et al. 2002, Eracleous et al. 2002). The steeper slope for the ellipticals could be indicative of a different mode of X-ray binary formation, or, perhaps more likely, an older XRB population that is in a later stage of X-ray evolution (e.g., see Grimm et al. 2002 and $\mathrm{Wu} 2001)$.

The total point source luminosity $\mathrm{L}_{X P}$ can be theoretically defined as

$$
L_{X P}^{\text {theor. }}=\int_{L_{\min }}^{L_{\max }} \operatorname{Ln}(L) d L \propto L_{\max }^{1-\gamma}-L_{\min }^{1-\gamma},
$$

where, for simplicity, we have assumed $\gamma \neq 1$. Here $n(L)$ is the differential luminosity function $\left[n(L)=N_{0} \gamma L^{-(\gamma+1)}\right.$ when $\left.\mathrm{N}(>\mathrm{L})=\mathrm{N}_{0} \mathrm{~L}^{-\gamma}\right]$, and $\mathrm{L}_{\min }$ and $\mathrm{L}_{\max }$ are the X-ray luminosities of the least and most luminous X-ray point sources in the galaxy, respectively. Since the slope of the cumulative XLF $\gamma<1$ for the Merger/Irr and spiral galaxies, $\mathrm{L}_{X P}$ is most sensitive to the upper limit $\mathrm{L}_{\max }$. Although $\mathrm{L}_{\min }$ and $\mathrm{L}_{\max }$ are different for each galaxy, in general, the ratio $\mathrm{L}_{\max } / \mathrm{L}_{\min } \gtrsim 10^{2}$ (see Figures 2 and 3 ), so that the $\mathrm{L}_{\max }$ term of the integral dominates over the $\mathrm{L}_{\text {min }}$ term by factors $\gtrsim 5.0$ and $\gtrsim 2.6$, for the Merger/Irr and spiral galaxies, respectively. This is quite interesting, since it implies that for galaxies with shallow XLFs, such as spiral galaxies, the most luminous point sources, such as the ULXs, dominated $\mathrm{L}_{X P}$. Furthermore, for galaxies with several sources above $10^{38} \mathrm{erg} \mathrm{s}^{-1}$, observational measurements of $\mathrm{L}_{X P}$ using very deep imaging data sensitive to very faint (e.g., $\gtrsim 10^{36} \mathrm{erg} \mathrm{s}^{-1}$ ) sources should give approximately the same value for $\mathrm{L}_{X P}$ as a shorter observation sensitive to sources above $\sim 10^{37} \mathrm{erg} \mathrm{s}^{-1}$. Therefore, we argue that the values we quote for $\mathrm{L}_{X P}$ in Table 3 are reliable for the spiral and Merger/Irr galaxies.

\footnotetext{
${ }^{2}$ We have taken special care to omit luminosity ranges on the lower end that could be incomplete due to poor luminosity sensitivity within the luminosity bin. The exact ranges used are listed in the notes to Table 4. We have also used a weighted least-squares technique to compute $\gamma$, which gives much less weight to the highest luminosity bins, compared to a simple least-squares technique. Our measurement of $\gamma$ may differ from other measurements reported in the literature (e.g. Kilgard et al. 2002), especially if different luminosity ranges were used, or the galaxy point-source lists are different.
} 


\subsection{X-ray Color-color diagrams}

X-ray color-color (CC) diagrams were constructed using net counts in three X-ray bands, soft $(\mathrm{S}-0.3-1.0 \mathrm{keV})$, medium $(\mathrm{M}-1.0-2.0 \mathrm{keV})$, and hard $(\mathrm{H}-2.0-8.0 \mathrm{keV})$. In our $\mathrm{CC}$ diagrams (Figure 4), we plot soft hardness $M S=(M-S) /(M+S)$ against hard hardness $H M=(H-M) /(H+M)$. The Poisson uncertainty in $H M$ and $M S$ is $\lesssim 0.30$ and $\lesssim 0.35$, respectively. ${ }^{3}$ Tabulated values of the net counts in each of the three bands, and hardness ratios, are listed in Table A1. In Figure 5, we show a grid of the expected locations of $\mathrm{X}$-ray sources, for a simple power-law model with foreground absorption. X-ray colors were simulated using the response of BI CCD 7. No foreground absorption corresponds to the lowest values of MS in the diagram, while increasing absorption pushes the source up, and eventually to the right, when the medium band $(1.0-2.0 \mathrm{keV})$ begins to be affected by absorption. In Figure 5, we show the areas of the CC diagram that would be occupied by three sample types of point sources found in galaxies: typical MW LMXBs, typical SMC HMXBs, which are much harder, and (spiral galaxy) ULXs, based on survey results from Church \& Balucinska-Church (2001), Yokogawa (2002), Foschini et al. (2002), and Roberts et al. (2002).

We first examine the CC diagrams of the elliptical galaxies in Figure 4, since they seem to be the least complex. Most of the point sources in the elliptical galaxies are consistent with the MW LMXBs (HM -0.5), with little or no $\left(\mathrm{N}_{H}<3 \times 10^{21} \mathrm{~cm}^{-2}\right)$ absorption. Some of the sources have harder X-ray colors (HM $\sim 0.25)$. One would not expect HMXBs in elliptical galaxies, and we offer two explanations. The harder sources are predominantly the faintest sources with a small number of total counts, so the uncertainties in their X-ray colors is larger than the other sources. Since the scatter of the points in the CC diagram appears nearly symmetric, this seems like the most likely explanation. A second consideration is that the MW LMXBs have lower X-ray luminosities $\left(\mathrm{L}_{X} \sim 10^{36}-10^{38} \mathrm{erg} \mathrm{s}^{-1}\right)$ than the elliptical galaxy sources $\left(\mathrm{L}_{X} \gtrsim 10^{38} \mathrm{erg} \mathrm{s}^{-1}\right)$, so there could be an additional smaller population of very-luminous harder-spectrum LMXBs in elliptical galaxies.

We next turn to the spiral galaxies (see Figure 4). There is noticeably more scatter in the CC diagram for the spiral galaxy sources than for the elliptical galaxy sources. The scatter is from the lower left to the upper right of the diagram, as one would expect for point sources with absorption columns ranging from negligible to $\sim 10^{23} \mathrm{~cm}^{-2}$. Most of the

\footnotetext{
${ }^{3}$ This estimate was determined empirically using the approximate Poisson error formula $\delta N \approx 1+$ $\sqrt{N+0.75}$ (e.g., Gehrels 1986) for the raw counts. For all of the sources with 20-100 net counts, the mean and standard deviation for $\Delta H M$ and $\Delta M S$ are $0.30 \pm 0.21$ and $0.34 \pm 0.57$, respectively. For sources with $>100$ net counts $\Delta H M=0.10 \pm 0.14$ and $\Delta M S=0.11 \pm 0.14$.
} 
sources in the spiral galaxies are not consistent with the hard-spectrum SMC HMXBs, which generally have $\mathrm{HM} \sim 0.25$. Their colors are more consistent with an absorbed power-law spectrum with $\Gamma \approx 1-2$. Even the most luminous sources (the ULXs, which are filled circles in Figure 4) in the spiral and Merger/Irr galaxies have soft $(\Gamma \sim 1-2)$ spectra. As mentioned in section 4.2 , the most luminous X-ray point sources dominate $\mathrm{L}_{X P}$ in the spiral and Merger/Irr galaxies, and, for most galaxies, this corresponds to point sources with $\mathrm{L}_{X}>$ $10^{38} \mathrm{erg} \mathrm{s}^{-1}$ (see XLFs in Figure 4). For convenience, in Figure 6, we show a CC diagram for only the sources with $\mathrm{L}_{X}>10^{38} \mathrm{erg} \mathrm{s}^{-1}$ in the spiral and Merger/Irr galaxies. From this plot, it is quite obvious that hard-spectrum $(\Gamma \approx 0.5-1)$ HMXBs are not dominating $\mathrm{L}_{X P}$ in these galaxies. The point-source luminosity $\mathrm{L}_{X P}$ in these two galaxy groups are ostensibly dominated by young (pop I) X-ray sources, so exactly what type of soft-spectrum sources are those that are plotted in Figure 6? Obviously, the ULXs (filled circles) will dominate $\mathrm{L}_{X P}$ when they are present; however, ULXs are generally present in only one of every $\sim 5$ disk galaxies (e.g. Sipior 2003). The sources with $\mathrm{L}_{X} \approx 10^{38}-10^{39} \mathrm{erg} \mathrm{s}^{-1}$ occupy essentially the same regions of the CC diagram as the ULXs, i.e., spectrally, there is no distinction between these two types of objects.

We can examine the spectral properties of well-studied (i.e. the nearest) luminous HMXBs to see if they are consistent with the hardness ratios of the spiral galaxy sources. In Table 5, we list all of the HMXBs with $\mathrm{L}_{X} \geq 10^{38} \mathrm{erg} \mathrm{s}^{-1}$, as listed in the XRB catalog of Guseinov et al. (2000; v0.1, URL: WWW.XRBC.ORG). These nine objects are the most luminous HMXBs in the MW and Magellanic Clouds. There are three Be/transient sources, three accretion-powered X-ray pulsars, two black hole candidates, and Cyg X-3, which is not well categorized. We also list the photon power law index $\Gamma$ from a simple absorbed powerlaw fit, even for those XRBs with complex X-ray spectral models (see Table 5 footnote).

Two of the three HMXB transients have hard spectra $(\Gamma \lesssim 1)$, similar to the SMC HMXBs plotted in Figure 5. The transient EXO 2030+375 is soft $(\Gamma \sim 1.8)$ during outburst, but hardens to $\Gamma \sim 1$ as it fades to $\sim 10^{37} \mathrm{erg} \mathrm{s}^{-1}$ (e.g., Reig \& Coe 1999). The accretionpowered NS HMXBs (SMC X-1, LMC X-4, and Cen X-3) have high and low states that are similar to those of the BH HMXB Cyg X-1 (high/soft and low/hard), but they have much harder spectra in their high states $\left(\Gamma_{\text {high }} \sim 1\right.$, compared to $\Gamma_{\text {high }} \sim 2.5$ for Cyg X-1). The two black-hole candidate (BHC) HMXBs LMC X-3 and LMC X-1 have high states with $\Gamma \sim$ $2-3$, similar to Cyg X-1. Cyg X-3 has a relatively soft spectrum $(\Gamma \sim 2)$ in its high state, which hardens to $\Gamma \sim 1$ in its low state, when the X-ray flux is reduced by a factor of $\sim 2$ (White \& Holt 1982).

Although a sample of nine luminous HMXBs is not a large sample, it is clear that the different types of very-luminous HMXBs have fairly unique spectral properties. Based on the 
CC diagram in Figure 6, the dominant X-ray sources in the spiral and Merger/Irr groups are generally inconsistent with accretion-powered NS HMXBs, which have $\Gamma \lesssim 1$. They are more consistent with BHC HMXBs, such as LMC X-3 and LMC X-1 in their soft state, with $\Gamma \sim$ $2-3$. It is not clear what fraction of $\mathrm{L}_{X P}$ from spiral and starbursting galaxies is from HMXB transients. If we could extrapolate from the MW and Magellanic Cloud HMXBs listed in Table 5, the accretion-powered (non-transient) HMXBs should dominate the soft-spectrum X-ray luminosity, since the X-ray luminosity of EXO $2030+375$ is only $\sim 1 / 6$ that of the combined X-ray luminosity of the "soft" accretion-powered HMXBs LMC X-3, LMC X-1, and Cyg X-3.

As we note in section 4.2, the pop II X-ray sources have a steeper XLF, and thus "normal" pop I BH HMXBs and pop I ULXs should dominate $\mathrm{L}_{X P}$ in the spiral and Merger/Irr galaxies. It is important to note that many of the ULXs may, in fact, be pop I BH HMXBs (e.g. King 2002), and, until ULXs are better understood, one should allow that there may be a substantial overlap between these two classes. In other words, ULXs are not necessarily distinct from the many known types of XRBs.

We conclude that the total point-source luminosity in spiral and starburst galaxies is not simply dominated by accretion powered, hard-spectrum NS HMXBs from the young stellar population. It is dominated by the very-luminous, soft-spectrum accretion-powered BH HMXBs, and soft-spectrum ULXs. These sources seem to have knowledge of (and are correlated with) both the current $\mathrm{SFR}$, as measured by $\mathrm{L}_{F I R}$ and $\mathrm{L}_{F I R+U V}$, and the mass of the galaxy, as measured by $\mathrm{L}_{B}$ and $\mathrm{L}_{K}$. Since the exact nature of ULXs are not well known and they will dominate $\mathrm{L}_{X P}$, if present, it is premature to say that the X-ray emission from the young stellar population is dominated by "high-mass X-ray binaries" (e.g. Grimm et al. 2003).

By inspecting the locations of the ULXs in the CC diagrams in Figure 4, one notices that, whereas the ULXs in the elliptical galaxies are clustered near HM $\sim-0.4$, the ULXs in the Merg/Irr and Spiral groups are slightly harder in HM. For example, there are no ULXs with HM $<-0.4$ in the spiral group. This could possibly be a result of obscuration by the gas in spirals, but this would require the ULXs to be highly obscured. As our simulations in Figure 5 (top left plot) show, absorption columns $\gtrsim 10^{22} \mathrm{~cm}^{-2}$ are required to increase HM by a factor $\gtrsim 0.4$. Alternatively, this could be a manifestation of two physically different types of ULXs (e.g., King 2002). 


\section{Discussion}

\subsection{Summary of Results}

In the previous section, we showed that the total point source X-ray luminosity $\mathrm{L}_{X P}$ is indeed correlated with properties of the host galaxy that are associated with the mass and SFR in the galaxy. Based on the XRB populations in our Galaxy, one might therefore suppose that the correlations merely represent an extrapolation of the total X-ray luminosity from the LMXB and HMXB populations, scaled to the galaxy mass and SFR (e.g., Grimm et al. 2002). Using X-ray color-color diagrams, we showed that the SFR component of $\mathrm{L}_{X P}$ is dominated by soft-spectrum BH HMXBs and ULXs, not hard-spectrum NS HMXBs. Since ULXs are not present in our Galaxy, X-ray studies of external galaxies are necessary to understand their effect on X-ray point source populations. Since we can actually measure the correlation between $\mathrm{L}_{X P}$ and either $\mathrm{L}_{K}$ or $\mathrm{L}_{F I R+U V}$, both the mass and SFR components of $\mathrm{L}_{X P}$ are significantly strong. Since the slope $\gamma$ of the XLFs is shallow for the spiral galaxies, the most luminous point sources (i.e., BH HMXBs and ULXs with $\mathrm{L}_{X} \gtrsim 10^{38}$ erg s s$^{-1}$ ) dominate $\mathrm{L}_{X P}$. We emphasize that the ULX distinction is purely based on X-ray luminosity and does not necessarily represent a unique, or homogeneous class of objects. For the spiral and Merger/Irr galaxies, we find no particular distinction between the X-ray colors of the ULXs and the less luminous point sources with $\mathrm{L}_{X} \approx 10^{38}-10^{39} \mathrm{erg} \mathrm{s}^{-1}$. As our CC diagrams show, the X-ray colors of the point sources are generally consistent with LMXBs or ULXs, not hard-spectrum NS HMXBs, such as accretion-powered X-ray pulsars. This is also true of the most luminous point sources (e.g., the ULXs).

\subsection{Multi-variate Correlations Between $\mathrm{L}_{X P}$ and Host Galaxy Luminosities}

We would like to deconvolve $\mathrm{L}_{X P}$ into two components, one from the older stellar population (including LMXBs), and another from the younger stellar population (including HMXBs). We assume the old and young components are directly related to the mass and SFR of the galaxy, respectively, and initially explore a simple relationship between $\mathrm{L}_{X P}$ and the host galaxy K and FIR+UV luminosities, which are reasonable proxies for the mass and SFR.

We use the simple linear relationship

$$
L_{X P}=A L_{K}+B L_{F I R+U V}
$$

where $A$ and $B$ are dimensionless constants. We have used $\chi^{2}$ fitting to estimate $\mathrm{A}$ and $\mathrm{B}$ and 
their uncertainties. ${ }^{4}$ The best fit values of $A$ and $B$ are listed in Table 6. Since star formation is negligible in the elliptical galaxies, we can also estimate $A$ directly from $\mathrm{L}_{X P}$ by ignoring the SFR term. If we omit the peculiar elliptical galaxy NGC 5128 (Cen A), which has ongoing star-formation, $\chi^{2}$-fitting yields $A=1.26_{-0.31}^{+0.30} \times 10^{-4}$. This value for ellipticals is $\sim 20 \%$ larger than that found from multi-variate $\chi^{2}$ fitting for samples involving Merger/Irr and Spiral galaxies (see Table 6), although the two values are consistent within the uncertainties. Since AGN emission in the K, FIR and FUV bands has not been subtracted from any of the host galaxy luminosities, we computed $A$ and $B$ for two subsamples of galaxies without AGNs, and arrive at a slightly smaller (larger) value for $A(B)$ than if AGNs are included. If the elliptical galaxies are also omitted from the sample, the best fit value of $A$ is also lower. We then suggest that the value of $A$ for early-type and elliptical galaxies is inherently different from that in late-type galaxies.

The fact that $A$ and $B$ are the same order of magnitude, and that $\mathrm{L}_{K}$ and $\mathrm{L}_{F I R+U V}$ are also the same order of magnitude, supports our earlier statement that both the old (mass) and young (SFR) component can contribute significantly to $\mathrm{L}_{X P}$.

We conclude, based on our best fit to the Merger/Irr plus spiral sample without AGNs, that the the point-source X-ray luminosity $\mathrm{L}_{X P}$ in Merger/Irr and spiral galaxies can be reliably estimated from:

$$
L_{X P}=(0.9 \pm 0.1) \times 10^{-4} L_{K}+(0.5 \pm 0.1) \times 10^{-4} L_{F I R+U V},
$$

and that $\mathrm{L}_{X P}$ for elliptical galaxies can be estimated from

$$
L_{X P}=(1.3 \pm 0.3) \times 10^{-4} L_{K}
$$

The robustness of this formula for the Merger/Irr and spiral galaxies can be seen in Figure 7 (top), where we plot $\mathrm{L}_{X P}$ against the estimated value using equation 4 . The Merger/Irr galaxies and Spiral galaxies are both in good agreement, although $\mathrm{L}_{X P}$ for the elliptical galaxies is under-estimated. As we $\operatorname{argue}$ in the next section, the larger $(\mathrm{M} / \mathrm{L})_{K}$ ratio for ellipticals (see Table 7 ) should likely cause the $A$ value for ellipticals and spirals to be inherently different.

We note that the reduced- $\chi^{2}, \chi_{\nu}^{2}$, for all of the fits (see Table 6) is highly sensitive to the estimated errors in the luminosity uncertainties, which are not well defined. Therefore,

\footnotetext{
${ }^{4}$ Errors for $\mathrm{L}_{K}$ and $\mathrm{L}_{F I R}$ were estimated from the errors in the flux measurements (see references in notes to Table 2). We estimated a $30 \%$ error for the FUV fluxes, due to uncertainties in fitting SEDs to the data (see section 3.3). We also experimented with other fitting methods (principal component analysis, simple least-squares, and robust weighted least-squares) and arrive at the same $A$ and $B$ values, within the $\chi^{2}$ uncertainties.
} 
we do not use $\chi_{\nu}^{2}$ as an absolute measure of the goodness of fit. We also note that the ranges of $A$ for spirals and ellipticals overlap, and we cannot rigorously show that the values of $A$ are different for ellipticals and spirals, although, as we show in the next section, the effect from including an $\mathrm{M} / \mathrm{L}$ correction is very noticeable.

\subsection{Multi-variate Correlations between $\mathrm{L}_{X P}$ and $\mathrm{M}$ and SFR}

We next write $\mathrm{L}_{X P}$ in terms of the mass $\mathrm{M}$ and SFR of the galaxy

$$
L_{X P}=\alpha M+\beta S F R
$$

where $\mathrm{L}_{X P}, \mathrm{M}$, and SFR have units $\operatorname{erg} \mathrm{s}^{-1}, \mathrm{M}_{\odot}$, and $\mathrm{M} \odot \mathrm{yr}^{-1}$, respectively, and $\alpha$ and $\beta$ are constants with the appropriate units.

We use the optical/NIR B-K color and the K-band luminosity (Table 2) to estimate the mass of the sample galaxies, and $\mathrm{L}_{F I R+U V}$ to estimate the SFR. Bell \& de Jong (2001) list correlation coefficients for $\mathrm{M} / \mathrm{L}$ ratios as a function of optical and NIR colors. We use the coefficients for their formation model with bursts. The SFR can be estimated from $\mathrm{L}_{F I R+U V}$ if we assume $\mathrm{L}_{F I R+U V}$ is a "corrected" IRAS FIR luminosity. We use a proportionality constant of $5.7 \times 10^{-44} \mathrm{M}_{\odot} \mathrm{yr}^{-1}\left(\mathrm{erg} \mathrm{s}^{-1}\right)^{-1}$, which includes a bolometric correction of 1.4 from the IRAS FIR band (Meurer et al. 1999). The resulting values for SFR, (M/L) $K$ and $M$ are listed in Table 7.

Again, we find $\alpha$ and $\beta$ and their uncertainties using minimum- $\chi^{2}$ techniques. The uncertainties in $\mathrm{M}$ and SFR were computed from the uncertainties in all of the luminosities (or magnitudes) used in computing $\mathrm{M}$ and SFR. In Table 6, we list the resulting values for the Merger/Irr plus spirals sample (omitting AGNs). We find that the mass-component constant $\alpha$ is slightly different for the elliptical and spiral samples, but this time $\alpha$ is smaller for the ellipticals, although both $\alpha$ values are consistent within their uncertainties. As can be seen in Figure 7 (bottom), with the M/L correction applied, the simulated value of $\mathrm{L}_{X P}$ now agrees better with the observed value. The simulated value of $\mathrm{L}_{X P}$ for the elliptical galaxies is now much closer to the observed value (compare top and bottom plots in Figure 7). This indicates that $\alpha=1.25 \times 10^{29} \mathrm{erg} \mathrm{s}^{-1} \mathrm{M}_{\odot}^{-1}$ is a universal constant for estimating the point-source X-ray luminosity from the older stellar population.

Thus, we find the following empirical relationship to hold for high-SFR/M Merger/Irr galaxies, spiral galaxies, and low-SFR/M elliptical galaxies:

$$
L_{X P}\left(\operatorname{erg~s}^{-1}\right)=(1.3 \pm 0.2) \times 10^{29} M\left(M_{\odot}\right)+(0.7 \pm 0.2) \times 10^{39} S F R\left(M_{\odot} y r^{-1}\right)
$$


As noted in the caption to Figure 7 , the observed values for $\mathrm{L}_{X P}$ for the two starburst galaxies NGC 4449 and NGC 4038/9 are in excess of the values predicted by Equations (4) and $(7)$, by factors of $\approx 4$ and $\approx 5$, respectively. Since $\mathrm{L}_{X P}$ is dominated by the $S F R$ component for starburst galaxies, an error in the $S F R$ by $\sim 4-5$ would be needed to "correct" the problem. This is unlikely. Poisson uncertainty in the number of high-luminosity sources (which dominate $\mathrm{L}_{X P}$ ) could possible explain the excess, but it is also possible that these two starburst galaxies have significantly high efficiency for forming luminous BH HMXBs and ULXs. It is interesting that NGC 4038/9 is a merging system and NGC 4449 has evidence for two physical counter-rotating systems within the galaxy (Sabbadin et al. 1984). More

extensive tests would be needed to determine if merger activity is really responsible for the abnormally high values of $\mathrm{L}_{X P}$ in these two galaxies.

\subsection{The Linearity of $\mathbf{L}_{X P}, M$, and $S F R$}

In order to test the linear form we have assumed in Equation (7), we performed a number of tests. A least-squares fit between $\log L_{X P}$ and $\log M$ for the eight elliptical galaxies in our sample with no star formation (NGC 5128 omitted) implies a best fit for $L_{X P} \propto M^{0.99}$ (r $=$ 0.89). A more rigorous test from a multi-variate fit to the sample of $18 \mathrm{Merg} / \mathrm{Irr}$ and spiral galaxies without AGN gives the best fit for the mass component of $\mathrm{L}_{X P} \propto M^{1.01}$ (See Figure 9). Therefore, we can argue with good confidence that the pop II component of $\mathrm{L}_{X P}$ is essentially linear in $M$. Similarly, a multi-variate fit to $L_{X P}=\alpha M+\beta S F R^{p_{S F R}}$ gives a best fit for $\mathrm{p}_{S F R}=1.06$, with a $90 \%$ confidence range of $0.85-1.25$ (for three free parameters: $\Delta \chi^{2}<6.25$; see Figure 9). We arrive at the a very similar result $\left(p_{S F R}=1.09\right)$ if we use only the five Merger/Irr galaxies, which have a very weak mass component, and fit $\log \left(\mathrm{L}_{X P}\right)$ against $\log (S F R)(r=0.94)$. Thus, within the uncertainties, we find $L_{X P}$ to be linear in both the mass and the star-formation rate.

\subsection{Nature of the ULXs}

As mentioned previously, we assume the mass component of $\mathrm{L}_{X P}$ is from point sources from an older stellar population (including LMXBs), and the SFR component is from point sources from a younger stellar population (including HMXBs). It is not known if ULXs, which can dominate $\mathrm{L}_{X P}$ (section 4.2), are predominantly associated with older or younger stellar population, or are a largely heterogeneous group (e.g., King 2002). Thus, they could contribute significantly in either component. We know that ULXs exist in elliptical galaxies (e.g. Colbert \& Ptak 2002). If LMXBs are formed in globular clusters (e.g. Kundu et al. 
2003), pop II ULXs might also be formed in the same environment. On the other hand, in the high-SFR galaxy pair NGC 4038/9 (The "Antennae"), $\mathrm{L}_{F I R}$ is very high and an extraordinarily large number of ULXs are found (Fabbiano, Zezas \& Murray 2001), suggesting that the ULXs in the Antennae are associated with the young stellar population. So what about ULXs in the normal spirals, in which old and young stellar populations (ie the $\mathrm{K}$ and FIR+UV luminosities) can be comparable?

In Figure 8, we plot the fraction of $\mathrm{L}_{X P}$ from the younger stellar population against galaxy morphological type. As expected, in late-type galaxies with high SFR/M ratios, the SFR term dominates, and in early-type galaxies, the mass term dominates. This plot also shows that the older population contributes $\approx 20-90 \%$ of $\mathrm{L}_{X P}$ in Merger/Irr and spiral galaxies. As described in section 4.2, since the slope of the XLF for these groups is shallow

$(\gamma \lesssim 1)$, the most luminous X-ray point sources dominate $\mathrm{L}_{X P}$. This would imply that, if the old and young components of the XLF have exactly the same shape, $\sim 20-90$ percent of the ULXs are from the older population. However, this is not likely to be the case since the XLFs for older point sources in the elliptical galaxies is much steeper than the XLFs for old+young point sources in the spirals, and many of the ULXs are from the younger population. However, it does suggest that type II (pop II) ULXs could have significant numbers in spiral galaxies. Since we now know that $\mathrm{L}_{X P}$ from the older stellar population is proportional to the galaxy mass $M$, we can estimate the fraction of type II ULXs in spirals by scaling the number found in ellipticals by the galaxy mass $M$. If we exclude NGC 5128 since it has ongoing star formation, the total mass of the remaining eight ellipticals is 1.06 $\times 10^{12} \mathrm{M}_{\odot}$, and eleven ULXs are found. This implies an expected $1.0 \times 10^{-11} \mathrm{ULXs} \mathrm{M}_{\odot}^{-1}$, with a Poisson error of $30 \%$. The total mass of all of the Merg/Irr and spiral galaxies is 6.6 $\times 10^{11} \mathrm{M}_{\odot}$, so we expect $\approx 5-9$ pop II ULXs to be found. A total of 32 ULXs were detected, implying that, on average, $\sim 15-25 \%$ of the ULXs in Merger/Irr and spiral galaxies are pop II ULXs. The same calculation for the Merger/Irr and spiral galaxy samples alone yield rates of $\sim 5-10 \%$ and $\sim 25-50 \%$ of pop II ULXs for the "high-SFR/M" Merger/Irr galaxies, and normal spirals, respectively.

\section{Summary and Conclusions}

We have used archival and proprietary Chandra ACIS data for 32 spiral, elliptical, and Merger/Irr (starburst) galaxies to study X-ray point source populations, and how they depend on properties of the host galaxy. A total of 1441 X-ray point sources are analyzed here, using the CPU-intensive XASSIST data reduction scripts (Ptak \& Griffiths 2003) to rigorously verify the validity of the point sources. 
The total point-source $\mathrm{X}$-ray $(0.3-8.0 \mathrm{keV})$ luminosity $\mathrm{L}_{X P}$ is well correlated with the B-band, K-band, and FIR+UV luminosities of spiral host galaxies, and is well correlated with the B-band and K-band luminosities for elliptical galaxies. This has been known for some time, and it suggests an intimate connection between $\mathrm{L}_{X P}$ and both the old and young stellar populations, for which $\mathrm{K}$ and FIR+UV luminosities are reasonable proxies for the galaxy mass $M$ and star-formation rate $S F R$.

We derive proportionality constants $\alpha=1.3 \times 10^{29} \mathrm{erg} \mathrm{s}^{-1} \mathrm{M}_{\odot}^{-1}$ and $\beta=0.7 \times 10^{39}$ $\operatorname{erg~s}^{-1}\left(\mathrm{M}_{\odot} \mathrm{yr}^{-1}\right)^{-1}$, which can be used to estimate the old and young components from $M$ and $S F R$, respectively.

The cumulative X-ray luminosity functions for the point sources have significantly different slopes. For the spiral and starburst galaxies, $\gamma \approx 0.6-0.8$, and for the elliptical galaxies, $\gamma \approx 1.4$. This implies that the most luminous point sources - those with $L_{X} \gtrsim 10^{38} \mathrm{erg} \mathrm{s}^{-1}$ - dominate $L_{X P}$ for the spiral and starburst galaxies. We find that $\mathrm{L}_{X P}$ depends linearly (within uncertainties) on both $M$ and $S F R$, for our sample galaxies $\left(M \lesssim 10^{11} \mathrm{M}_{\odot}\right.$ and $\left.S F R \lesssim 10 \mathrm{M}_{\odot} \mathrm{yr}^{-1}\right)$.

Most of the point sources have X-ray colors that are consistent with soft-spectrum $(\Gamma \sim 1-2)$ low-mass X-ray binaries (XRBs), accretion-powered black-hole high-mass (BH HMXBs), or Ultra-Luminous X-ray sources (ULXs a.k.a. IXOs). We rule out hard-spectrum neutron-star HMXBs (e.g. accretion-powered X-ray pulsars) as contributing much to $\mathrm{L}_{X P}$. Thus, for spirals, $\mathrm{L}_{X P}$ is dominated by ULXs and BH HMXBs. We find no discernible difference between the X-ray colors of ULXs $\left(\mathrm{L}_{X} \geq 10^{39} \mathrm{erg} \mathrm{s}^{-1}\right)$ in spiral galaxies and point sources with $\mathrm{L}_{X} \approx 10^{38}-10^{39} \mathrm{erg} \mathrm{s}^{-1}$, suggesting ULXs in spiral galaxies could be predominantly BH HMXBs. This would not be surprising, since ULXs are defined purely by their X-ray luminosity, and not by their accretion mode, or type of companion star. More work on ULXs is needed to test whether most are simply a special mode of a "normal" stellar-mass BH XRB (e.g. King 2002), or exotic accreting intermediate-mass BH systems (e.g. Colbert \& Mushotzky 1999).

We estimate that $\gtrsim 20 \%$ of all ULXs found in spirals originate from the older (pop II) stellar populations, indicating that many of the ULXs that have been found in spiral galaxies are in fact pop II ULXs, like those in elliptical galaxies.

We thank the anonymous referee for many helpful suggestions that helped to improve the quality of the paper. We are grateful to T. Jarrett for providing 2MASS $\mathrm{K}_{s}$ magnitudes from the Large Galaxy Atlas before publication. We thank A. Zezas, T. Roberts, T. Yaqoob and A. Prestwich for helpful discussions, and T. Budavari for expert help with SED fitting. EJMC acknowledges support from NASA grant NAG 5-11670. 


\section{A. Tabulation of X-ray Point-source Properties}

As mentioned in section 1, uniform data processing of all of the datasets is important

for large samples of galaxies. We list in Table A1 some of the X-ray properties for each of the 1441 point sources in the 32 galaxies in our sample. 


\section{REFERENCES}

Audley, M. D. et al. 1996, ApJ, 457, 397

Bell, E. F., \& de Jong, R. S. 2001, ApJ, 550, 212

Bolzonella, M., Miralles, J.-M., \& Pello, R. 2000, A\&A, 363, 476

Calzetti, D., Kinney, A. L., \& Storchi-Bergmann, T. 1994, ApJ, 429, 582

Church, M. J., \& Balucinska-Church, M. 2001, A\&A, 369, 915

Colbert, E. J. M., \& Ptak, A. F. 2002, ApJS, 143, 25

Colbert, E. J. M., \& Mushotzky, R. F. 1999, ApJ, 519, 89

Corbet, R. H. D., Charles, P. A., Southwell, K. A., \& Smale, A. P. 1997, ApJ, 476, 833

Dickey, J. M., \& Lockman, F. J. 1990, ARA\&A, 28, 215

de Vaucouleurs, G., de Vaucouleurs, A., Corwin, H. G., Buta, R. J., Paturel, G., \& Fouque, P. 1991, Third Reference Catalog of Bright Galaxies (RC3; New York: Springer-Verlag)

Eracleous, M., Shields, J. C., Chartas, G., \& Moran, E. C. 2002, ApJ, 565, 108

Fabbiano, G. 1989, ARA\&A, 27, 87

Fabbiano, G., Gioia, I. M., \& Trinchieri, G. 1988, ApJ, 324, 749

Fabbiano, G., Zezas, A., \& Murray, S. S. 2001, ApJ, 554, 1035

Foschini, L., et al. 2002, A\&A, 392, 817

Fullmer, L., \& Lonsdale, C. 1989, Cataloged Galaxies and Quasars in the IRAS Survey (JPL Pub. D-1932, Version 2, Appendix B)

Gehrels, N. 1986, ApJ, 303, 336

Grimm, H.-J., Gilfanov, M., \& Sunyaev, R. 2002, A\&A, 391, 923

Grimm, H.-J., Gilfanov, M., \& Sunyaev, R. 2003, MNRAS, 339, 793

Guseinov, O. H., Saygac, A. T., Allakhverdiev, A., Caliskan, H., Ozdemir, S., Yerli, S. K., \& Ankay, A. 2000, AstL, 26, 725

Helfand, D. J., \& Moran, E. C. 2001, ApJ, 554, 27 
Ho, L. C., Filippenko, A. V., \& Sargent, W. L. W. 1997, ApJS, 112, 315

Irwin, J. A., Bregman, J. L., \& Sarazin, C. L. 2002a, in "X-rays at Sharp Focus: Chandra Science," ASP Conf. Ser. Vol. 262, ed. E. M. Schlegel and S. Vrtilek (San Francisco: ASP), p. 157

Irwin, J. A., Sarazin, C. L., \& Bregman, J. L. 2002b, ApJ, 570, 152

Jarrett, T. H., Chester, T., Cutri, R., Schneider, S., \& Huchra, J., 2003, AJ, 125, 525

Kennicutt, R. 1998, ARA\&A, 36, 189

Kilgard, R. E., Kaaret, P., Krauss, M. I., Prestwich, A. H., Raley, M. T., \& Zezas, A. 2002, ApJ, 573, 138

King, A. R. 2002, MNRAS, 335, L13

Kinney, A. L., Calzetti, D., Bohlin, R. C., McQuade, K., Storchi-Bergmann, T., \& Schmitt, H. R. 1996, ApJ, 467, 38

Knapp, J. 1994, priv. comm. to NED (correction to data from Knapp et al. 1989)

Knapp, G. R., Guhathakurta, P., Kim, D.-W., \& Jura, M. A. 1989, ApJS, 70, 329

Kundu, A., Maccarone, T. J., Zepf, S. E., \& Puzia, T. H. 2003, ApJ, 589, L81

Marcum, P. M., et al. 2001, ApJS, 132, 129

Meurer, G. R., Heckman, T. M., \& Calzetti, D. 1999, ApJ, 521, 64

Miller, M. C., \& Colbert, E. J. M. 2003, IJMPD, in press

Moshir, M., et al. 1992, Explanatory Supplement to the IRAS Faint Source Catalog, version 2, JPL D-10015 (Pasadena: JPL)

Moran, E. C., \& Lehnert, M. D. 1997, ApJ, 478, 172

Nowak, M. A., Wilms, J., Heindl, W. A., Pottschmidt, K., Dove, J. B., \& Begelman, M. C. 2001, MNRAS, 320, 316

Parmar FIX-check-2paper et al. 1989

Paul, B., Nagase, F., Endo, T., Dotani, T., Yokogawa, J., \& Nishiuchi, M. 2002, ApJ, 579, 411 
Primini, E. A., Forman, W., \& Jones, C. 1993, ApJ, 410, 615

Prugniel, Ph., \& Héraudeau, Ph. 1998, A\&AS, 128, 299

Ptak, A., \& Griffiths, R. 2003, in Astronomical Data Analysis Software \& Systems XII, eds. H. Payne, R. Jedrzejewski, \& R. Hook, ASP conference series vol. 295, 465

Ptak, A., Serlemitsos, P., Yaqoob, T., Mushotzky, R., \& Tsuru, T. 1997, AJ, 113, 1286

Ranalli, P., Comastri, A., \& Setti, G. 2003, A\&A, 399, 39

Reig, P., \& Coe, M. J. 1999, MNRAS, 302, 700

Rice, W., et al. 1988, ApJS, 68, 91

Rifatto, A., Longo, G., \& Capaccioli, M. 1995, A\&AS, 114, 527

Roberts, T. P., Goad, M. R., Ward, M. J., Warwick, R. S., \& Lira, P. 2002, in "New Visions of the X-ray Universe in the XMM-Newton and Chandra Era," (ESTEC: The Netherlands) (astro-ph/0202017)

Sabbadin, F., Ortolani, S., \& Bianchini, A., 1984, A\&A, 131, 1

Sandage, A., \& Tammann, G. A. 1981, Revised Shapley-Ames Catalog of Bright Galaxies (Carnegie Institute of Washington Publication 635)

Sarazin, C. L., Irwin, J. A., \& Bregman, J. N. 2001, ApJ, 556, 533

Sipior, M. 2003, Ph. D. Thesis, The Pennsylvania State University

Soifer, B. T., Boehmer, L., Neugebauer, G., \& Sanders, D. B. 1989, AJ, 98, 766

Swartz, D. A., Ghosh, K. K., McCollough, M. L., Pannuti, T. G., Tennant, A. F., \& Wu, K. 2003, ApJS, 144, 213

Thronson, H. A., Jr., Hunter, D. A., Telesco, C. M., Decher, R., \& Harper, D. A. 1987, ApJ, 317, 180

Tully, R. B. 1988, Nearby Galaxies Catalog (Cambridge: Cambridge Univ. Press)

White, N. E., \& Holt, S. S. 1982, ApJ, 257, 318

Wu, K. 2001, PASA, 18, 443

Yokogawa, J., 2002, Ph. D. Thesis, Kyoto University 
Yokogawa, J., Torii, K., Kohmura, T., \& Koyama, K. 2001, PASJ, 53, 227 
Figures are not included here. Please download full manuscript from the following website for version with figures. Sorry. This URL is also listed in the notes to the astro-ph abstract, so you may be able to just click on it there.

http://www.pha.jhu.edu/ colbert/chps_accepted.ps 
Table 1. Observational Data

\begin{tabular}{|c|c|c|c|c|c|}
\hline $\begin{array}{c}\text { Galaxy } \\
\text { Name } \\
(1)\end{array}$ & $\begin{array}{c}\text { Other } \\
\text { Name } \\
(2)\end{array}$ & $\begin{array}{c}\text { ObsID } \\
(3)\end{array}$ & $\begin{array}{c}\text { CCDs } \\
(4)\end{array}$ & $\begin{array}{c}\text { Exp. Time } \\
\text { (ks) } \\
(5)\end{array}$ & $\begin{array}{l}\mathrm{N} \\
(6)\end{array}$ \\
\hline NGC 1569 & & 782 & 7 & 85.1 & 12 \\
\hline NGC 3034 & M82 & 361 & $0 / 1 / 2 / 3$ & $33.2 / 32.8 / 33.1 / 33.0$ & 27 \\
\hline NGC $4038 / 9$ & Antennae & 315 & $6 / 7$ & $72.1 / 70.2$ & 65 \\
\hline NGC 4449 & & 2031 & 7 & 26.2 & 22 \\
\hline NGC 5253 & & 2032 & 7 & 46.7 & 10 \\
\hline NGC 253 & & 969 & $2 / 3 / 6 / 7$ & 13.9/13.9/13.9/11.8 & 49 \\
\hline NGC 628 & M74 & 2057 & $6 / 7$ & $46.2 / 44.7$ & 60 \\
\hline NGC 1291 & & 2059 & $6 / 7$ & $11.2 / 12.0$ & 56 \\
\hline NGC 2681 & & 2061 & 7 & 78.3 & 17 \\
\hline NGC 3079 & & 2038 & 7 & 26.3 & 17 \\
\hline NGC 3184 & & 804 & 7 & 37.1 & 45 \\
\hline NGC 3628 & & 2039 & 7 & 54.8 & 32 \\
\hline NGC 4244 & & 942 & 7 & 48.1 & 3 \\
\hline NGC 4258 & M106 & 350 & $6 / 7$ & $14.0 / 13.9$ & 26 \\
\hline NGC 4314 & & 2062 & 7 & 8.3 & 12 \\
\hline NGC 4579 & M58 & 807 & 7 & 32.8 & 8 \\
\hline NGC 4631 & & 797 & $6 / 7$ & $59.1 / 57.6$ & 25 \\
\hline NGC 4736 & M94 & 808 & 7 & 47.3 & 28 \\
\hline NGC 4945 & & 864 & $6 / 7$ & $35.2 / 5.1$ & 36 \\
\hline NGC 5194/5 & M51a/b & 354 & $6 / 7$ & 14.8 & 52 \\
\hline NGC 5236 & M83 & 793 & $6 / 7$ & $50.9 / 48.2$ & 84 \\
\hline NGC 5457 & M101 & 934 & $2 / 3 / 5 / 6 / 7$ & $98.1 / 98.1 / 96.3 / 98.2 / 95.6$ & 140 \\
\hline NGC 6503 & & 872 & 7 & 9.1 & 7 \\
\hline NGC 1395 & & 799 & 3 & 22.2 & 30 \\
\hline NGC 1399 & & 319 & 7 & 55.5 & 100 \\
\hline NGC 3379 & M105 & 1587 & 7 & 31.0 & 35 \\
\hline NGC 4374 & M84 & 803 & 7 & 28.3 & 28 \\
\hline NGC 4472 & M49 & 321 & $6 / 7$ & $39.2 / 33.8$ & 78 \\
\hline NGC 4636 & & 323 & 7 & 45.2 & 37 \\
\hline NGC 4649 & M60 & 785 & $6 / 7$ & $36.7 / 22.3$ & 98 \\
\hline NGC 4697 & & 784 & 7 & 39.0 & 69 \\
\hline NGC 5128 & Cen A & 316 & $0 / 1 / 2 / 3$ & $35.5 / 35.3 / 35.4 / 35.5$ & 133 \\
\hline
\end{tabular}

Notes on table columns: (1,2) Galaxy Names; (3) Chandra Observation ID; (4) ACIS CCD numbers in galaxy FOV for which point sources were detected; (5) Exposure time for CCDs listed in column 4; (6) Number of X-ray point sources detected within galaxy FOV (see section 3 for details). 
Table 2. Galaxy Properties

\begin{tabular}{|c|c|c|c|c|c|c|c|c|c|}
\hline $\begin{array}{c}\text { Galaxy } \\
\text { Name } \\
(1)\end{array}$ & $\begin{array}{c}\text { Distance } \\
(\mathrm{Mpc}) \\
(2)\end{array}$ & $\begin{array}{c}\text { Morph. } \\
\text { Type } \\
(3)\end{array}$ & $\begin{array}{c}\text { AGN } \\
\text { Type } \\
(4)\end{array}$ & $\begin{array}{c}\mathrm{L}_{B} \\
(\log \mathrm{erg} / \mathrm{s}) \\
(5)\end{array}$ & $\begin{array}{c}\mathrm{L}_{K} \\
(\log \mathrm{erg} / \mathrm{s}) \\
(6)\end{array}$ & $\begin{array}{c}\mathrm{L}_{F I R} \\
(\log \mathrm{erg} / \mathrm{s}) \\
(7)\end{array}$ & $\begin{array}{c}\mathrm{L}_{U V} \\
(\log \mathrm{erg} / \mathrm{s}) \\
(8)\end{array}$ & $\begin{array}{c}\frac{L_{F I R}}{L_{B}} \\
(9)\end{array}$ & $\begin{array}{c}\frac{L_{F I R+U V}}{L_{K}} \\
(10)\end{array}$ \\
\hline NGC 1569 & 1.6 & $\mathrm{Sm}$ & & 42.2 & 41.3 & 41.8 & 40.3 & 0.4 & 3.6 \\
\hline NGC 3034 & 5.2 & I0 & & 43.4 & 43.6 & 44.2 & 41.7 & 6.0 & 4.0 \\
\hline NGC $4038 / 9$ & 21.7 & $\mathrm{Sc} / \mathrm{Sc}$ (tides) & & 44.3 & 43.8 & 44.1 & 43.4 & 0.7 & 2.2 \\
\hline NGC 4449 & 3.0 & $\mathrm{Sm}$ & & 42.5 & 42.0 & 42.4 & 42.1 & 0.7 & 3.6 \\
\hline NGC 5253 & 3.2 & I0 & & 42.4 & 41.7 & 42.2 & 41.9 & 0.7 & 5.5 \\
\hline NGC 253 & 3.0 & $\mathrm{Sc}$ & & 43.7 & 43.5 & 43.6 & 41.8 & 0.9 & 1.4 \\
\hline NGC 628 & 9.7 & $\mathrm{Sc}$ & & 43.6 & 43.1 & 43.2 & 42.8 & 0.4 & 1.7 \\
\hline NGC 1291 & 8.6 & $\mathrm{SBa}$ & & 43.7 & 43.6 & 42.1 & 42.1 & 0.02 & 0.06 \\
\hline NGC 2681 & 13.3 & $\mathrm{Sa}$ & & 43.4 & 43.3 & 42.9 & 41.9 & 0.3 & 0.5 \\
\hline NGC 3079 & 15.0 & Sc pec: & $\mathrm{S} 2$ & 43.7 & 43.5 & 43.8 & 42.2 & 1.3 & 2.4 \\
\hline NGC 3184 & 8.7 & $\mathrm{Sc}$ & & 43.3 & 42.9 & 42.8 & 42.6 & 0.3 & 1.3 \\
\hline NGC 3628 & 7.7 & $\mathrm{Sbc}$ & & 43.6 & 43.3 & 43.3 & 42.4 & 0.5 & 1.1 \\
\hline NGC 4244 & 3.1 & Scd & & 42.8 & 41.8 & 41.6 & 41.6 & 0.06 & 1.3 \\
\hline NGC 4258 & 6.8 & $\mathrm{Sb}$ & S1.9 & 43.8 & 43.5 & 43.0 & 42.4 & 0.2 & 0.4 \\
\hline NGC 4314 & 9.7 & $\mathrm{SBa}$ & & 43.0 & 43.0 & 42.4 & 41.6 & 0.2 & 0.3 \\
\hline NGC 4579 & 20.3 & Sab & S1.9/L1.9 & 44.0 & 44.0 & 43.3 & 42.7 & 0.2 & 0.2 \\
\hline NGC 4631 & 6.9 & $\mathrm{Sc}$ & & 43.8 & 43.1 & 43.3 & 42.9 & 0.3 & 2.3 \\
\hline NGC 4736 & 4.3 & RSab & & 43.3 & 43.2 & 42.9 & 42.3 & 0.4 & 0.6 \\
\hline NGC 4945 & 5.2 & $\mathrm{Sc}$ & AGN & 44.0 & 43.7 & 43.8 & 41.7 & 0.6 & 1.4 \\
\hline NGC $5194 / 5$ & 7.7 & $\mathrm{Sbc} / \mathrm{SB}$ & $\mathrm{S} 2$ & 43.9 & 43.8 & 43.3 & 43.1 & 0.2 & 0.5 \\
\hline NGC 5236 & 4.7 & $\mathrm{SBc}$ & & 43.7 & 43.5 & 43.2 & 42.5 & 0.4 & 0.7 \\
\hline NGC 5457 & 5.4 & $\mathrm{Sc}$ & & 43.7 & 43.1 & 43.3 & 43.4 & 0.4 & 3.2 \\
\hline NGC 6503 & 6.1 & $\mathrm{Sc}$ & & 43.1 & 42.7 & 42.4 & 42.0 & 0.2 & 0.8 \\
\hline NGC 1395 & 22.7 & E2 & & 44.0 & 43.9 & 41.6 & 42.3 & 0.003 & 0.03 \\
\hline NGC 1399 & 19.3 & E1 & & 43.9 & 44.0 & $41.3^{\mathrm{a}}$ & 42.5 & 0.002 & 0.03 \\
\hline NGC 3379 & 8.1 & E0 & & 43.3 & 43.3 & $<40.3$ & 41.7 & $<0.001$ & $<0.02$ \\
\hline NGC 4374 & 16.8 & $\mathrm{E} 1$ & & 44.0 & 44.0 & 42.0 & 42.4 & 0.01 & 0.04 \\
\hline NGC 4472 & 16.8 & $\mathrm{E} 1 / \mathrm{S} 0$ & $\mathrm{~S} 2::$ & 44.3 & 44.3 & $<41.1$ & 42.8 & $<0.0006$ & $<0.03$ \\
\hline NGC 4636 & 14.6 & $\mathrm{E} 0 / \mathrm{S} 0$ & & 43.7 & 43.7 & $41.2^{\mathrm{a}}$ & 42.4 & 0.003 & 0.05 \\
\hline NGC 4649 & 14.9 & S0 & & 44.0 & 44.1 & 42.0 & 42.5 & 0.01 & 0.03 \\
\hline NGC 4697 & 16.5 & E6 & & 43.9 & 43.9 & 42.0 & 42.3 & 0.01 & 0.04 \\
\hline NGC 5128 & 4.9 & $\mathrm{~S} 0+\mathrm{S}$ pec & AGN & 44.0 & 43.8 & 43.4 & 42.5 & 0.3 & 0.4 \\
\hline
\end{tabular}

Notes on columns: (1) Galaxy name; (2) Distance to galaxy (see section 2); (3) Galaxy morphological type, from the RSA galaxy catalog (Sandage \& Tammann 1981); (4) AGN type, as listed in Ho et al. (1997). We have additionally classified NGC 4945 and NGC 5128 as AGN, due to hard X-ray spectra properties, and nuclear jet, respectively. Blank entries denote LINER 1.9/2, transition, and H II classifications from Ho et al., or no evidence for AGN activity in the NED notes for that galaxy. (5) Galaxy B-band luminosity $\nu L \nu$, calculated using $\mathrm{B}_{T}^{0}$ (or $\mathrm{m}_{B}^{0}$ for NGC 4038/9) magnitudes from RC3; (6) Galaxy $\mathrm{K}_{s}$-band luminosity $\nu L \nu$, calculated from $20 \mathrm{mag} / \mathrm{sq}$. arcsec isophotal magnitudes from the 2MASS Large Galaxy Atlas (Jarrett et al. 2003); (7) Galaxy FIR luminosities, calculated from IRAS fluxes using the method of Fullmer \& Lonsdale (1989). IRAS fluxes were taken preferentially from Moshir et al. (1997, as listed in NED), Soifer et al. (1989), Rice et al. (1988), Knapp (1994), Knapp et al. (1989), and Thronson et al. (1987); (8) Galaxy far-ultraviolet luminosity (see section 3.3); (9) Ratio of FIR to B-band luminosity; (10) Ratio of FIR+UV to $\mathrm{K}_{s}$ luminosity.

${ }^{a}$ Only upper limits were available for the $60 \mu \mathrm{m}$ IRAS flux for NGC 1399 and the $100 \mu \mathrm{m}$ flux for NGC 4636 (Knapp 1994). The $\mathrm{L}_{F I R}$ value listed here was calculated using $50 \%$ of the quoted upper limit. The corresponding uncertainties in $\mathrm{L}_{F I R}$ due to this estimate are 0.05 and 0.08 dex for NGC 1399 and NGC 4636, respectively. 
Table 3. Galaxy X-ray Properties

\begin{tabular}{|c|c|c|c|c|c|c|c|c|}
\hline \multirow{2}{*}{$\begin{array}{c}\text { Galaxy } \\
\text { Name } \\
(1)\end{array}$} & \multirow{2}{*}{$\begin{array}{c}\mathrm{N}_{H}(\mathrm{Gal}) \\
\left(\log \mathrm{cm}^{-2}\right) \\
(2)\end{array}$} & \multirow{2}{*}{$\begin{array}{c}\mathrm{L}_{X}(\text { limit }) \\
(\log \mathrm{erg} / \mathrm{s}) \\
(3)\end{array}$} & \multirow{2}{*}{$\begin{array}{c}\mathrm{L}_{X P} \\
(\log \mathrm{erg} / \mathrm{s}) \\
(4)\end{array}$} & \multicolumn{2}{|c|}{ fraction $\geq 10^{38}$} & \multicolumn{2}{|c|}{ fraction $\geq 10^{39}$} & \multirow{2}{*}{$\begin{array}{c}\log \left(\frac{L_{X P}}{L_{K}}\right) \\
(9)\end{array}$} \\
\hline & & & & $\begin{array}{c}\mathrm{L}_{X P} \\
(5)\end{array}$ & $\begin{array}{c}\mathrm{N} \\
(6)\end{array}$ & $\begin{array}{c}\mathrm{L}_{X P} \\
(7)\end{array}$ & $\begin{array}{l}\mathrm{N} \\
(8)\end{array}$ & \\
\hline NGC 1569 & 21.4 & 35.4 & 37.9 & 0.00 & $0.00(0)$ & 0.00 & $0.00(0)$ & -3.4 \\
\hline NGC 3034 & 20.6 & 36.9 & 40.0 & 0.97 & $0.56(15)$ & 0.61 & $0.11(3)$ & -3.6 \\
\hline NGC $4038 / 9$ & 20.6 & 37.7 & 40.8 & 0.99 & $0.82(53)$ & 0.77 & $0.22(14)$ & -3.0 \\
\hline NGC 4449 & 20.1 & 36.3 & 39.0 & 0.66 & $0.14(3)$ & 0.00 & $0.00(0)$ & -3.0 \\
\hline NGC 5253 & 20.6 & 36.2 & 38.0 & 0.00 & $0.00(0)$ & 0.00 & $0.00(0)$ & -3.6 \\
\hline NGC 253 & 20.2 & 36.7 & 39.4 & 0.65 & $0.12(6)$ & 0.00 & $0.00(0)$ & -4.1 \\
\hline NGC 628 & 20.7 & 37.2 & 39.6 & 0.54 & $0.15(9)$ & 0.00 & $0.00(0)$ & -3.6 \\
\hline NGC 1291 & 20.3 & 37.6 & 39.7 & 0.65 & $0.23(13)$ & 0.00 & $0.00(0)$ & -3.8 \\
\hline NGC 2681 & 20.4 & 37.2 & 39.7 & 0.93 & $0.53(9)$ & 0.59 & $0.12(2)$ & -3.6 \\
\hline NGC 3079 & 19.9 & 37.7 & 39.6 & 0.84 & $0.41(7)$ & 0.43 & $0.06(1)$ & -3.8 \\
\hline NGC 3184 & 20.0 & 37.1 & 39.4 & 0.44 & $0.07(3)$ & 0.00 & $0.00(0)$ & -3.5 \\
\hline NGC 3628 & 20.3 & 36.8 & 39.5 & 0.71 & $0.09(3)$ & 0.63 & $0.03(1)$ & -3.9 \\
\hline NGC 4244 & 20.2 & 36.1 & 37.6 & 0.00 & $0.00(0)$ & 0.00 & $0.00(0)$ & -4.2 \\
\hline NGC 4258 & 20.1 & 37.3 & 39.6 & 0.83 & $0.42(11)$ & 0.00 & $0.00(0)$ & -3.8 \\
\hline NGC 4314 & 20.3 & 37.9 & 39.0 & 0.66 & $0.33(4)$ & 0.00 & $0.00(0)$ & -3.9 \\
\hline NGC 4579 & 20.4 & 37.9 & 40.2 & 1.00 & $1.00(8)$ & 0.87 & $0.25(2)$ & -3.8 \\
\hline NGC 4631 & 20.1 & 36.7 & 39.5 & 0.86 & $0.20(5)$ & 0.53 & $0.04(1)$ & -3.6 \\
\hline NGC 4736 & 20.2 & 36.4 & 39.6 & 0.90 & $0.29(8)$ & 0.55 & $0.07(2)$ & -3.6 \\
\hline NGC 4945 & 21.2 & 37.6 & 39.8 & 0.88 & $0.39(14)$ & 0.39 & $0.06(2)$ & -3.8 \\
\hline NGC 5194/5 & 20.2 & 37.4 & 40.0 & 0.84 & $0.33(17)$ & 0.33 & $0.04(2)$ & -3.8 \\
\hline NGC 5236 & 20.6 & 36.5 & 39.8 & 0.69 & $0.17(14)$ & 0.18 & $0.01(1)$ & -3.7 \\
\hline NGC 5457 & 20.1 & 36.3 & 39.8 & 0.66 & $0.06(8)$ & 0.25 & $0.01(1)$ & -3.4 \\
\hline NGC 6503 & 20.6 & 37.4 & 39.1 & 0.85 & $0.43(3)$ & 0.00 & $0.00(0)$ & -3.6 \\
\hline NGC 1395 & 20.3 & 38.4 & 40.2 & 1.00 & $1.00(30)$ & 0.49 & $0.10(3)$ & -3.7 \\
\hline NGC 1399 & 20.1 & 37.6 & 40.4 & 0.91 & $0.74(74)$ & 0.23 & $0.03(3)$ & -3.6 \\
\hline NGC 3379 & 20.4 & 37.1 & 39.5 & 0.73 & $0.23(8)$ & 0.00 & $0.00(0)$ & -3.8 \\
\hline NGC 4374 & 20.4 & 37.8 & 40.2 & 0.98 & $0.86(24)$ & 0.63 & $0.04(1)$ & -3.8 \\
\hline NGC 4472 & 20.2 & 37.7 & 40.3 & 0.96 & $0.87(68)$ & 0.14 & $0.03(2)$ & -4.0 \\
\hline NGC 4636 & 20.3 & 37.5 & 39.6 & 0.69 & $0.49(18)$ & 0.00 & $0.00(0)$ & -4.1 \\
\hline NGC 4649 & 20.3 & 37.8 & 40.2 & 0.80 & $0.56(55)$ & 0.07 & $0.01(1)$ & -3.9 \\
\hline NGC 4697 & 20.3 & 37.6 & 40.1 & 0.86 & $0.58(40)$ & 0.11 & $0.01(1)$ & -3.8 \\
\hline NGC 5128 & 20.9 & 36.9 & 40.1 & 0.66 & $0.19(25)$ & 0.15 & $0.01(1)$ & -3.8 \\
\hline
\end{tabular}

Notes on columns: (1) Galaxy Name; (2) Neutral Hydrogen absorption column of the Milky Way, from the FTOOLS NH program (Dickey \& Lockman 1990); (3) Limiting X-ray luminosity calculated using PIMMS, assuming a detection of 10 net counts, a power-law model with photon index $\Gamma=1.7$, and the Galactic absorption listed in column 2. Since most of the galaxies were centered on CCD7 and most of the point sources were positioned on that chip, we assumed the spectral response for CCD7, and the exposure time for CCD7 listed in Table 1, except for the following galaxies: NGC 3034 (CCD0), NGC 1395 (CCD3), and NGC 5128 (CCD0). (4) Observed X-ray point-source luminosity, from all point sources (see Table 1, column 6). Note that nuclear and near-nuclear (within 5 " of the NED astrometric position) X-ray point sources have been omitted for the following galaxies: NGC 3079, NGC 4258, NGC 4579, NGC 4945, NGC 5194, NGC 4374, and NGC 5128. Artificial X-ray sources due to saturation along the CCD readout direction ("readout stripes") have been omitted for NGC 4579 and NGC 5128. X-ray sources along the "jet" in NGC 5128 have also been omitted. (5,6) Fraction of $\mathrm{L}_{X P}$ and $\mathrm{N}$ (the number of X-ray point sources listed in Table 1) from sources with $\mathrm{L}_{X} \geq 10^{38} \mathrm{erg} \mathrm{s}^{-1}$. The number of sources with $\mathrm{L}_{X} \geq 10^{38} \mathrm{erg} \mathrm{s}^{-1}$ is listed in parentheses in column 6 ; $(7,8)$ Same as columns $(5,6)$ except for $10^{39} \mathrm{erg} \mathrm{s}^{-1}$; (9) Ratio of $\mathrm{L}_{X} P$ to galaxy $\mathrm{K}_{s}$ band luminosity from Table 2 . 
Table 4. Slopes of Cumulative X-ray Luminosity Functions

\begin{tabular}{|c|c|c|c|c|}
\hline $\begin{array}{c}\text { Galaxy } \\
\text { Name } \\
(1)\end{array}$ & $\begin{array}{c}\bar{L}_{X}(\min ) \\
(\log \mathrm{erg} / \mathrm{s}) \\
(2)\end{array}$ & $\begin{array}{c}\bar{L}_{X}(\max ) \\
(\log \mathrm{erg} / \mathrm{s}) \\
(3)\end{array}$ & $\begin{array}{l}\mathrm{N} \\
(4)\end{array}$ & $\begin{array}{c}\gamma \\
(5)\end{array}$ \\
\hline NGC 1569 & 36.0 & 37.5 & 5 & 0.44 \\
\hline NGC 3034 & 37.5 & 39.5 & 20 & 0.57 \\
\hline NGC $4038 / 9$ & 38.0 & 40.0 & 53 & 0.63 \\
\hline NGC 4449 & 37.0 & 38.4 & 12 & 0.70 \\
\hline NGC 5253 & 36.5 & 37.7 & 8 & 0.92 \\
\hline NGC 253 & 37.0 & 38.6 & 36 & 0.75 \\
\hline NGC 628 & 37.5 & 38.6 & 38 & 1.11 \\
\hline NGC 1291 & 37.7 & 38.9 & 31 & 1.16 \\
\hline NGC 2681 & 37.8 & 39.2 & 10 & 0.65 \\
\hline NGC 3079 & 37.5 & 39.2 & 17 & 0.80 \\
\hline NGC 3184 & 37.5 & 38.7 & 20 & 1.15 \\
\hline NGC 3628 & 37.0 & 39.3 & 28 & 0.82 \\
\hline NGC 4244 & 36.6 & 37.5 & 3 & 0.46 \\
\hline NGC 4258 & 37.5 & 38.8 & 23 & 0.79 \\
\hline NGC 4314 & 37.7 & 38.2 & 7 & 1.20 \\
\hline NGC 4579 & 38.5 & 40.1 & 5 & 0.47 \\
\hline NGC 4631 & 37.0 & 39.2 & 23 & 0.69 \\
\hline NGC 4736 & 37.2 & 39.1 & 19 & 0.55 \\
\hline NGC 4945 & 37.6 & 39.1 & 22 & 0.70 \\
\hline NGC 5194/5 & 37.7 & 39.1 & 29 & 0.78 \\
\hline NGC 5236 & 37.5 & 39.0 & 34 & 0.91 \\
\hline NGC 5457 & 37.0 & 39.2 & 68 & 0.85 \\
\hline NGC 6503 & 37.5 & 38.7 & 4 & 0.37 \\
\hline NGC 1395 & 38.3 & 39.7 & 22 & 1.05 \\
\hline NGC 1399 & 38.0 & 39.4 & 79 & 1.26 \\
\hline NGC 3379 & 37.8 & 39.0 & 12 & 1.07 \\
\hline NGC 4374 & 38.0 & 38.6 & 26 & 1.16 \\
\hline NGC 4472 & 38.5 & 39.2 & 19 & 1.63 \\
\hline NGC 4636 & 38.0 & 38.6 & 22 & 2.36 \\
\hline NGC 4649 & 38.0 & 39.0 & 62 & 1.58 \\
\hline NGC 4697 & 38.0 & 39.1 & 39 & 1.34 \\
\hline NGC 5128 & 38.0 & 39.2 & 28 & 1.28 \\
\hline
\end{tabular}

Notes on columns: (1) Galaxy Name; $(2,3)$ The slope $\gamma$ was computed using a weighted least-squares algorithm with logarithmic bins in X-ray luminosity. $\bar{L}_{X}(\min )$ and $\bar{L}_{X}(\max )$ are the luminosities corresponding to (the center of) the minimum and maximum bin used to compute $\gamma$. The bins have width of 0.1 dex. $\bar{L}_{X}(\min )$ was chosen to maximize the range in $\mathrm{L}_{X P}$ without including very low $\mathrm{L}_{X P}$ values, where a completeness problem may exist (see Figures 2 and 3). (4) Total number of X-ray point sources used in the fit; (5) Slope $\gamma$ of the cumulative XLF 
Table 5. X-ray Spectral Properties of Luminous HMXBs

\begin{tabular}{lcccccc}
\hline \hline $\begin{array}{c}\text { Name } \\
(1)\end{array}$ & $\begin{array}{c}\text { Type } \\
(2)\end{array}$ & $\begin{array}{c}\mathrm{L}_{X} / 10^{38} \mathrm{erg} \mathrm{s}^{-1} \\
(3)\end{array}$ & $\begin{array}{c}\text { Instr. } \\
(4)\end{array}$ & $\begin{array}{c}\Gamma \\
(5)\end{array}$ & $\begin{array}{c}\text { Ref. } \\
(6)\end{array}$ & $\begin{array}{c}\text { Notes } \\
(7)\end{array}$ \\
\hline SMC X-2 & $\mathrm{Be} / \mathrm{T}$ & 1 & ASCA & 0.7 & 1 & outburst \\
A 0538-66 & $\mathrm{Be} / \mathrm{T}$ & 6 & ASCA & 1.16 & 2 & outburst \\
EXO 2030+375 & $\mathrm{Be} / \mathrm{T}$ & 1 & EXOSAT & 1.83 & 3 & outburst \\
\hline SMC X-1 & AcPXP & 6 & ASCA & $1.05^{\dagger}$ & 4 & high state \\
LMC X-4 & AcPXP & 6 & ASCA & $0.70^{\dagger}$ & 4 & high state \\
LMC X-3 & BHC & 3 & RXTE & $1.8^{\dagger}$ & 5 & high state \\
LMC X-1 & BHC & 2 & RXTE & $2.9^{\dagger}$ & 5 & high state \\
Cen X-3 & AcPXP & 1 & BBXRT & 1.1 & 6 & high state \\
\hline Cyg X-3 & $?$ & 1 & & $\sim 2$ & 7 & high state \\
& & 0.4 & & $\sim 1$ & 7 & low state \\
\hline
\end{tabular}

Notes on columns: $(1,2)$ Source name, and HMXB type. X-ray transients (all in outburst) are listed first (all are Be/Transients), then accretion-powered X-ray pulsars (AcPXPs) and accretion-powered black-hole candidates (BHCs); (3) X-ray luminosity, as listed in Guseinov et al. (2000); (4) Instrument used for determining $\Gamma$; (5) Photon index $\Gamma$ for simple powerlaw fit; (6) Reference for $\Gamma$ ([1] Yokogawa et al. 2001, [2] Corbet et al. 1997, [3] Parmar et al. 1989, [4] Paul et al. 2002, [5] Nowak et al. 2001, [6] Audley et al. 1996, [7] White \& Holt 1982); (7) Notes on HMXB state during observation

$\dagger$ Since these sources have complex X-ray spectra, requiring both a hard (power-law) and a soft component in when observed with reasonably $\mathrm{S} / \mathrm{N}, \Gamma$ was determined by simulating a Chandra ACIS-S X-ray spectrum with XSPEC, then re-fitting the simulated data with a simple absorbed power-law model. For SMC X-1 and LMC X-4, we used models III from Tables 1 and 3 from Paul et al. (2002, respectively) to simulate the spectra, and, for LMC X-3 and LMC X-1, respectively, we used models from Tables 2 and 3 of Nowak et al. (2001). 
Table 6. Proportionality Constants for $\mathrm{L}_{X P}$

\begin{tabular}{ccccccl}
\hline \hline $\mathrm{A}$ & $\mathrm{B}$ & $\chi_{\nu}^{2}$ & $\alpha$ & $\beta$ & $\chi_{\nu}^{2}$ & \multicolumn{1}{c}{$\begin{array}{c}\text { Sample Description (No. of galaxies) } \\
(1)\end{array}$} \\
& $(2)$ & $(3)$ & $(4)$ & $(5)$ & $(6)$ & \\
\hline $1.26_{-0.31}^{+0.30}$ & $\ldots$ & 0.81 & $0.88_{-0.21}^{+0.22}$ & $\ldots$ & 0.90 & Elliptical galaxies only (8) \\
$1.11_{-0.11}^{+0.11}$ & $0.31_{-0.10}^{+0.10}$ & 4.61 & $1.05_{-0.11}^{+0.11}$ & $0.74_{-0.15}^{+0.15}$ & 3.79 & All galaxies with FIR flux measurements (30) \\
$1.01_{-0.14}^{+0.14}$ & $0.44_{-0.13}^{+0.14}$ & 5.53 & $1.09_{-0.14}^{+0.14}$ & $0.86_{-0.19}^{+0.19}$ & 4.40 & All galaxies with FIR flux measurements, excepting AGN (24) \\
$0.93_{-0.15}^{+0.15}$ & $0.49_{-0.13}^{+0.14}$ & 7.21 & $1.25_{-0.17}^{+0.17}$ & $0.74_{-0.18}^{+0.20}$ & 5.56 & Only M/I and Spiral galaxies, no AGN (18) \\
\hline
\end{tabular}

Notes on columns: $(1,2)$ Dimensionless A and B constants for correlation of $\mathrm{L}_{X P}$ with host galaxy luminosities $\mathrm{L}_{K}$ and $\mathrm{L}_{F I R+U V}$, respectively (see section 5.3, equation 1); (3) Reduced- $\chi^{2}$ for the fit $=\chi^{2} /$ (no. of galaxies - no. of parameters); $(4,5)$ Constants $\alpha$ and $\beta$, for correlation with $\mathrm{M}$ and SFR (see equation 2). Units are $\operatorname{erg~s}^{-1} \mathrm{M}_{\odot}^{-1}$ and $\operatorname{erg~s}^{-1}\left(\mathrm{M}_{\odot} \mathrm{yr}^{-1}\right)^{-1}, \operatorname{respectively}$ (6) Reduced- $\chi^{2}$ for the fit; (7) Description of the galaxy sample used in the calculation (number of galaxies in parentheses)

All quoted uncertainties are $90 \%$ confidence for one parameter free $\left(\Delta \chi^{2}=2.7\right)$. For example, errors in $A$ were determined by fixing $B$ at its minimum- $\chi^{2}$ value and scanning over $A$. 
Table 7. Inferred Properties of Host Galaxies

\begin{tabular}{|c|c|c|c|}
\hline $\begin{array}{c}\text { Galaxy } \\
\text { Name } \\
(1)\end{array}$ & $\begin{array}{c}\mathrm{SFR} \\
\left(\mathrm{M}_{\left.\odot \mathrm{yr}^{-1}\right)}\right. \\
(2)\end{array}$ & $\begin{array}{c}\log (M / L)_{K} \\
(\odot) \\
(3)\end{array}$ & $\begin{array}{c}\text { Mass } \\
\log \left(\mathrm{M}_{\odot}\right) \\
(4)\end{array}$ \\
\hline NGC 1569 & 0.037 & -0.6 & 7.9 \\
\hline NGC 3034 & 9.3 & -0.1 & 10.8 \\
\hline NGC $4038 / 9$ & 8.3 & -0.4 & 10.7 \\
\hline NGC 4449 & 0.21 & -0.4 & 8.9 \\
\hline NGC 5253 & 0.14 & -0.5 & 8.4 \\
\hline NGC 253 & 2.4 & -0.3 & 10.5 \\
\hline NGC 628 & 1.3 & -0.4 & 10.0 \\
\hline NGC 1291 & 0.13 & -0.2 & 10.7 \\
\hline NGC 2681 & 0.47 & -0.3 & 10.3 \\
\hline NGC 3079 & 4.0 & -0.3 & 10.5 \\
\hline NGC 3184 & 0.56 & -0.4 & 9.8 \\
\hline NGC 3628 & 1.3 & -0.3 & 10.3 \\
\hline NGC 4244 & 0.047 & -0.7 & 8.4 \\
\hline NGC 4258 & 0.69 & -0.3 & 10.4 \\
\hline NGC 4314 & 0.16 & -0.2 & 10.1 \\
\hline NGC 4579 & 1.4 & -0.2 & 11.1 \\
\hline NGC 4631 & 1.6 & -0.5 & 9.8 \\
\hline NGC 4736 & 0.58 & -0.2 & 10.4 \\
\hline NGC 4945 & 3.6 & -0.3 & 10.6 \\
\hline NGC 5194/5 & 1.8 & -0.2 & 10.8 \\
\hline NGC 5236 & 1.2 & -0.3 & 10.5 \\
\hline NGC 5457 & 2.5 & -0.5 & 10.0 \\
\hline NGC 6503 & 0.20 & -0.4 & 9.6 \\
\hline NGC 1395 & $\ldots$ & -0.2 & 11.0 \\
\hline NGC 1399 & $\ldots$ & -0.1 & 11.2 \\
\hline NGC 3379 & $\ldots$ & -0.1 & 10.5 \\
\hline NGC 4374 & $\ldots$ & -0.2 & 11.1 \\
\hline NGC 4472 & $\ldots$ & -0.1 & 11.5 \\
\hline NGC 4636 & $\ldots$ & -0.1 & 10.9 \\
\hline NGC 4649 & $\ldots$ & -0.1 & 11.2 \\
\hline NGC 4697 & $\ldots$ & -0.2 & 11.0 \\
\hline NGC 5128 & $1.7^{\mathrm{a}}$ & -0.2 & 10.9 \\
\hline
\end{tabular}

Notes on columns: (1) Galaxy name; (2) Star formation rate, estimated from $\mathrm{L}_{F I R+U V}$ (see text section 5.2); (3) Stellar mass-to-light ratio for the $\mathrm{K}$ band, in solar units, calculated from B-K (Table 2). See text section 5.2 for details; (4) Stellar mass, calculated using $\mathrm{L}_{K}$ from Table 2, and $(\mathrm{M} / \mathrm{L})_{K}$ from column 3.

${ }^{\text {a }}$ Since the FIR emission from elliptical galaxies is not necessarily dominated by re-radiated emission from hot stars, it may not be a good predictor of the SFR in elliptical galaxies. However, since NGC 5128 does have ongoing star formation, we have estimated a SFR for it. 
Table A1. Properties of the X-ray Point Sources

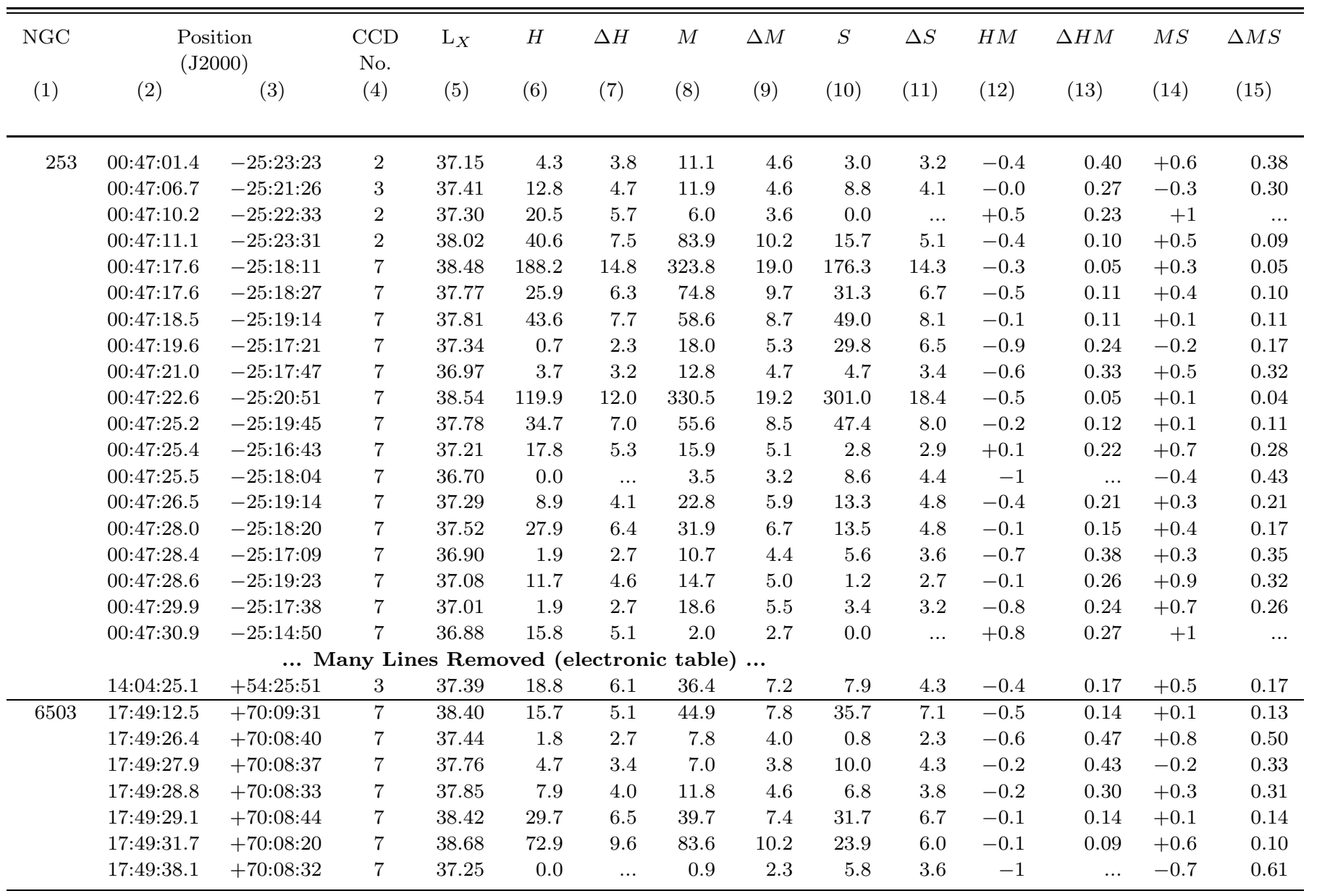

Notes on columns: (1) Host Galaxy NGC number (see Table 1); (2,3) X-ray position in J2000 coordinates; (4) ACIS CCD number. CCDs 5 and 7 are back-illuminated, and the others are front-illuminated (see section 3.1); (5) Logarithm of the $0.3-8.0 \mathrm{keV} \mathrm{X}-\mathrm{ray}$ luminosity, assuming galaxy distances listed in Table 2 and exposure times listed in Table 1. See section 3.1 for details about assumed spectrum; (6-11) Net observed counts and uncertainties for the Hard, Medium and Soft bands $(H, M$, and $S$ ), as described in section 4.3; (12-15) Hardness ratios $H M=(H-S) /(H+S)$ and $M S=(M-S) /(M+S)$ for BI CCD 7, and corresponding uncertainties, for front-illuminated CCD number 7 , as described in section 3.1. Uncertainties are not listed for hardness ratios when no net counts were detected in one of the bands. 